University of Louisville

ThinkIR: The University of Louisville's Institutional Repository

Electronic Theses and Dissertations

$12-2019$

\title{
Exploring normalization of deviance and examining factors that predict culture of safety in the operating room.
}

\author{
M. Imelda Wright \\ University of Louisville
}

Follow this and additional works at: https://ir.library.louisville.edu/etd

Part of the Nursing Administration Commons, and the Perioperative, Operating Room and Surgical Nursing Commons

\section{Recommended Citation}

Wright, M. Imelda, "Exploring normalization of deviance and examining factors that predict culture of safety in the operating room." (2019). Electronic Theses and Dissertations. Paper 3339.

https://doi.org/10.18297/etd/3339

This Doctoral Dissertation is brought to you for free and open access by ThinkIR: The University of Louisville's Institutional Repository. It has been accepted for inclusion in Electronic Theses and Dissertations by an authorized administrator of ThinkIR: The University of Louisville's Institutional Repository. This title appears here courtesy of the author, who has retained all other copyrights. For more information, please contact thinkir@louisville.edu. 


\title{
EXPLORING NORMALIZATION OF DEVIANCE AND EXAMINING FACTORS THAT PREDICT CULTURE OF SAFETY IN THE OPERATING ROOM
}

\author{
By \\ M. Imelda Wright \\ Diploma in Nursing, Catherine McCauley School of Nursing, Mercy Hospital, Cork, \\ Ireland, 1992 \\ B.S.N., McKendree University, 2008 \\ M.S.N., McKendree University, 2015
}

\begin{abstract}
A Dissertation
Submitted to the Faculty of the School of Nursing of the University of Louisville In Partial Fulfillment of the Requirements

For the Degree of
\end{abstract}

Doctor of Philosophy in Nursing

School of Nursing University of Louisville

Louisville, Kentucky

December 2019 
Copyright 2019 by M. Imelda Wright

All rights reserved 



\title{
EXPLORING NORMALIZATION OF DEVIANCE AND EXAMINING FACTORS THAT PREDICT CULTURE OF SAFETY IN THE OPERATING ROOM
}

\author{
By \\ M. Imelda Wright \\ Diploma in Nursing, Catherine McAuley School of Nursing, Mercy Hospital, Cork, \\ Ireland, 1992 \\ B.S.N., McKendree University, 2008 \\ M.S.N., McKendree University, 2015
}

A Dissertation Approved on

October 31, 2019

By the following Dissertation Committee

\begin{tabular}{c} 
Dissertation Director \\
Barbara Polivka, PhD, RN, FAAN \\
\hline Said Abusalem, PhD, RN \\
\hline Paul Clark, PhD, RN, MA \\
\hline
\end{tabular}

Janet Odom-Foren, PhD, RN, CPAN, FAAN 


\section{DEDICATION}

This dissertation is reflective of a long journey of hard work, learning, and growth, both personally and professionally.

I dedicate my work to my parents David and Sheila O' Leary, who were two of the hardest working people that I have ever known. Although neither of them are alive today, I am certain that they would have supported me on this journey. While I am proud of my accomplishment, I fully recognize that I was privileged to receive tremendous life opportunities that offered a prestigious path of education to me, opportunities that were never offered to my parents. As I celebrate the attainment of this monumental life goal, I am humbled as I remember them because I realize that I will never live up to the tremendous life successes that they achieved as evidenced by their lives of humility, dedication to each other and their family, and nurturing a home so that nine children could love, respect, and honor each other long after they are gone. I am blessed to have had them as parents and role models.

I also dedicate this unique body of work to my daughter Sarah, who is my greatest joy. My hope is that she can learn from having watched her mother over the last several years that any life goal is reachable with enough hard work, dedication, and passion. While there is no expectation for her to follow my path, I have high hopes that Sarah will attain great success on her own life path. May she be blessed with tenacity and strength to overcome adversity as it comes and the knowledge that she is supported and loved dearly. 


\section{ACKNOWLEDGEMENT}

Although I am immensely proud of the work and commitment that I gave to my PhD path, I am deeply humbled with the knowledge that none of this would be possible without the dedicated faculty members at the University of Louisville who guided me along the way.

In particular, Dr. Barbara Polivka has been my rock of encouragement during the past four years. She consistently encouraged me to be all that I could be and pushed me beyond my comfort zone on several occasions, which always made me stronger and more empowered. Her dedication and commitment to her students is unmatched, and I recognize how deeply fortunate I have been to have had her as my mentor, committee chairperson, and role model.

I also acknowledge my other committee members, Dr. Said Abusalem, Dr. Paul Clark, and Dr. Jan Odom-Forren who were always available to me for support, advice, and guidance. Finally, I acknowledge Dr. Becky Christian, the director of the $\mathrm{PhD}$ program at the U of L School of Nursing, who has always been a constant source of guidance, encouragement, and friendship as I ventured on the $\mathrm{PhD}$ journey. 


\section{ABSTRACT \\ EXPLORING NORAMLIZATION OF DEVIANCE AND EXAMINING FACTORS THAT PREDICT CULTURE OF SAFETY IN THE OPERATING ROOM

\author{
M. Imelda Wright
}

October 31, 2019

Patient safety in the operating room is a prodigious responsibility of OR nurses as the OR environment is complex and laden with risk, which increases surgical patients' risk for adverse events. The dissertation presents the topic of patient safety in the operating room (OR), the concept of normalization of deviance, and a mixed-methods study with an exploratory sequential design that involved three phases.

The dissertation comprises of five chapters. Chapter One presents an introductory overview of the salience of patient safety as well as its relevance to the unique environment of the OR. Chapter Two presents a concept analysis of normalization of deviance, which may be defined as the adoption of substandard practices, which typically occurs after a period of absence of adverse events. Chapter Three presents Phase 1, which is the qualitative study that sought to examine primary OR safety concerns, explore the occurrence of normalization of deviance, and examine barriers and facilitators to the adherence to safety standards. Ten interviews with OR nurses were conducted. Chapter Four presents Phase 2, which is the quantitative study that sought to examine the strength and association of relationships between the length of OR nurse experience, OR nurse engagement and OR culture of safety . Data were collected from 96 OR nurses from an online platform. Chapter Five presents Phase 3 of the study that synthesized the 
findings of engagement, and OR culture of safety. Data were collected from 96 OR nurses from an online platform. Chapter Five presents Phase 3 of the study that synthesized the findings of both the previous phases.

The study findings demonstrate that normalization of deviance occurs in the OR. Barriers to adherences to safety standards and reasons for normalization of deviance include productivity pressure, complacency related to length of experience, social pressures, generalized complacency, and negative acculturation. Facilitators to the adherence to safety standards and protective factors against normalization of deviance include OR nurse engagement and supportive managerial relationships with OR nurses. In addition, OR nurse engagement was a significant predictor of OR culture of safety. It is imperative to acknowledge and mitigate barriers to the adherence to patient safety. It is also necessary to promote and encourage facilitators to the adherence to safety standards, such as OR nurse engagement and supportive managerial relationships with OR nurses. 


\section{TABLE OF CONTENTS}

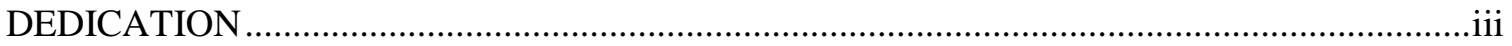

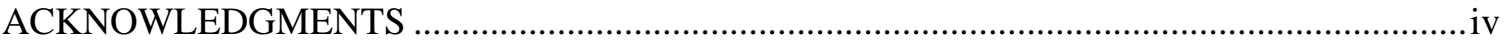

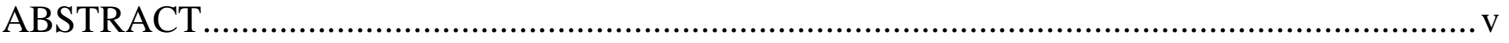

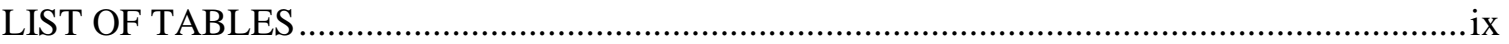

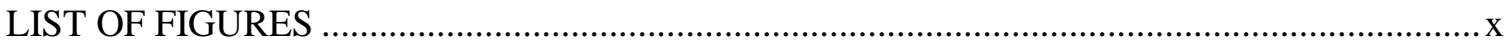

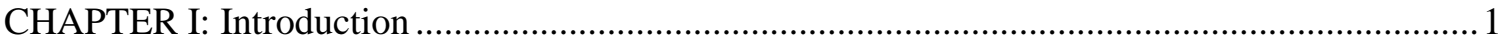

CHAPTER II: Concept Analysis: Normalization of Deviance................................

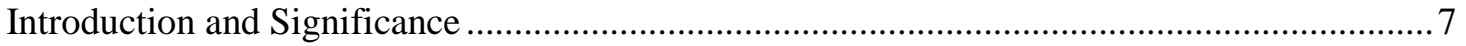

Purpose

Uses of the concept 'Normalization of Deviance'............................................................. 11

Defining the Attributes of Normalization of Deviance..................................................... 13

Model 'Normalization of Deviance' Case …................................................................... 14

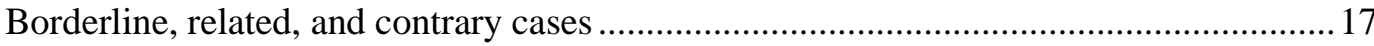

Antecedents and Consequences to Normalization of Deviance .........................................19

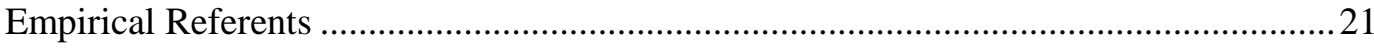

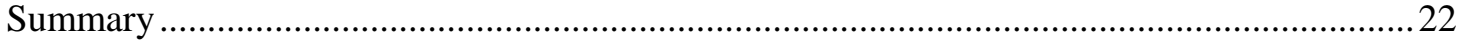

CHAPTER III: Exploring Normalization of Deviance in the Operating Room ..........................25

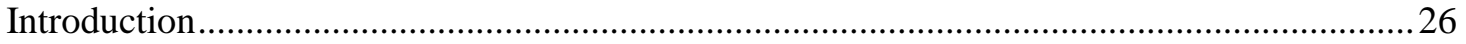

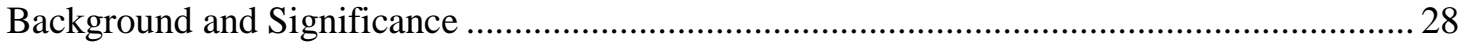

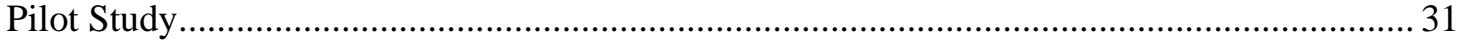

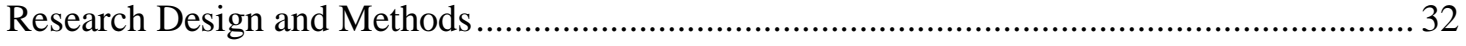

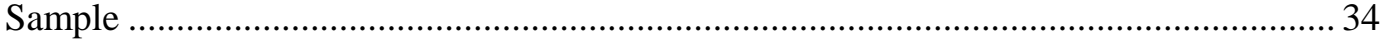

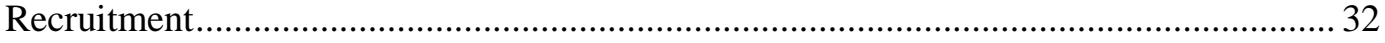

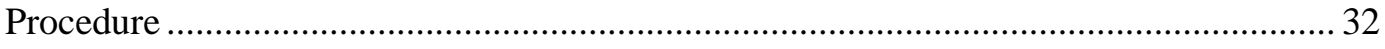

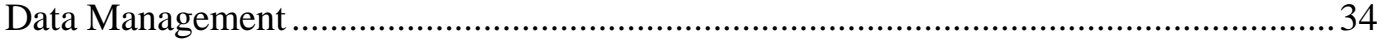

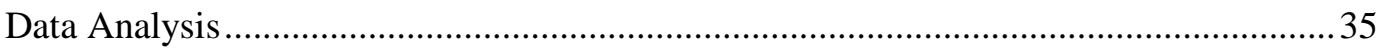

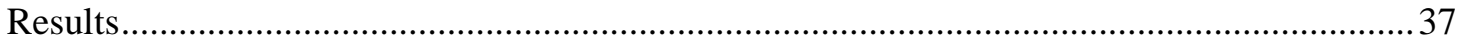

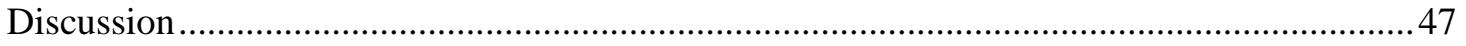




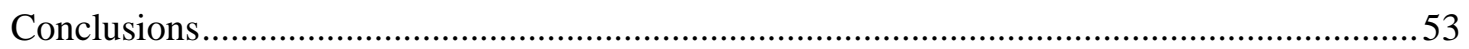

CHAPTER IV: Examining Factors that Predict Culture of Safety in the Operating Room ..........65

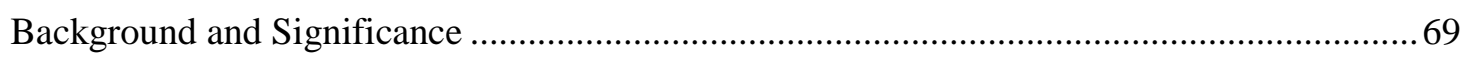

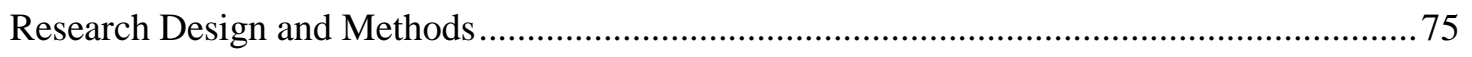

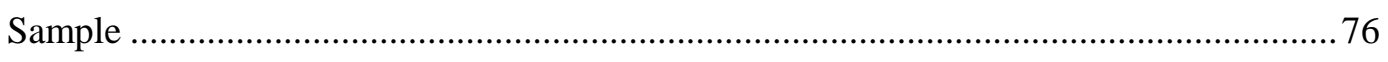

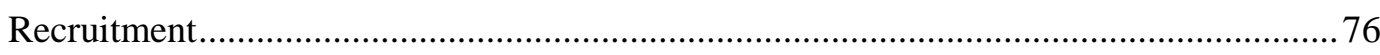

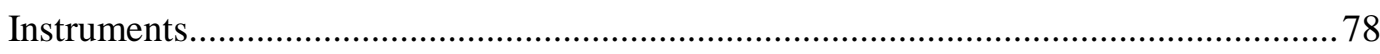

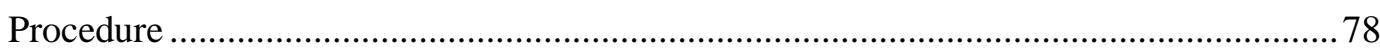

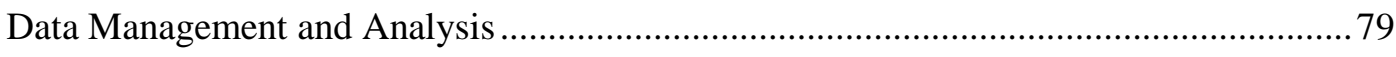

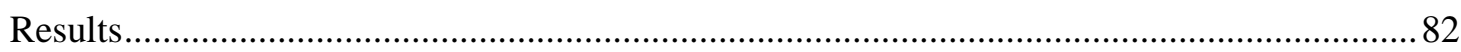

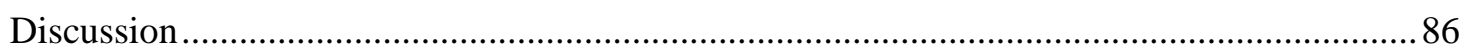

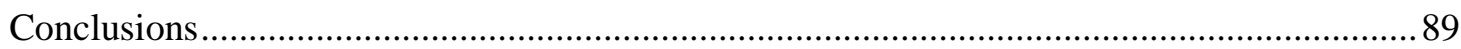

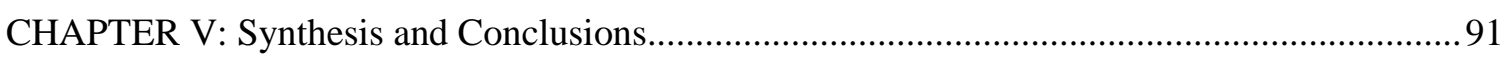

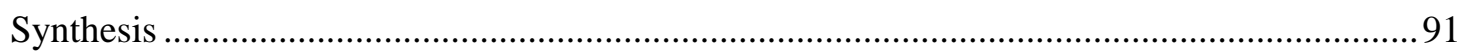

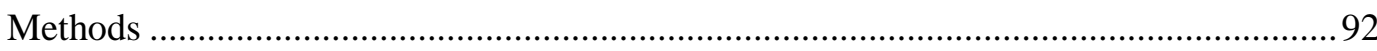

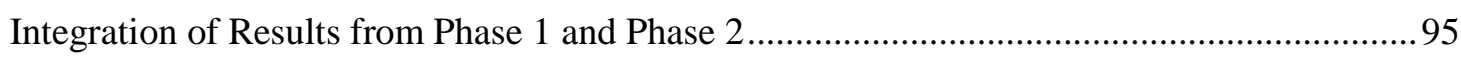

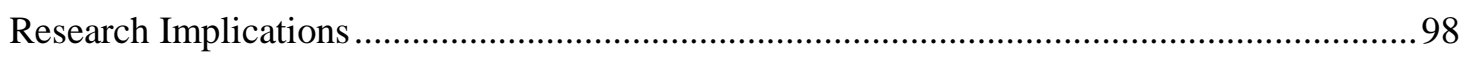

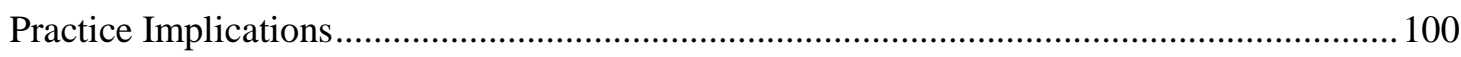

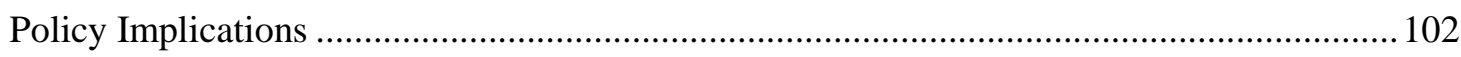

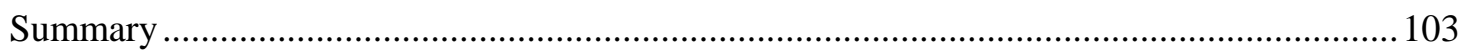

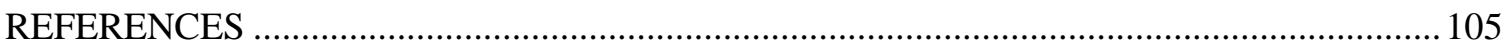

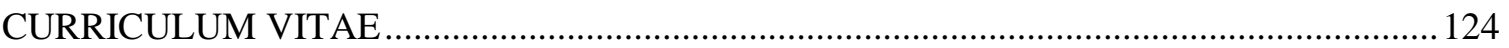




\section{LIST OF TABLES}

TABLES

PAGE

1. Descriptive Characteristics of Participants (Qualitative Study)...................37

2. Interview Questions................................................. 55

3. Primary safety concerns as described by OR nurses..........................56

4. Quotes supporting the occurrence of Normalization of Deviance in the OR ......58

5. Quotes supporting perceived Barriers to the adherence of safety standards/Reasons for Normalization of Deviance.............................................. 59

6. Quotes supporting perceived Facilitators to adherence to safety standards/Protective factors against Normalization of Deviance.................................62

7. Quotes representing Unsupportive Managerial Relationships with OR nurses

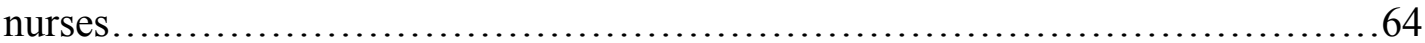

8. Descriptive Characteristics of Participants (Quantitative Study) .................82

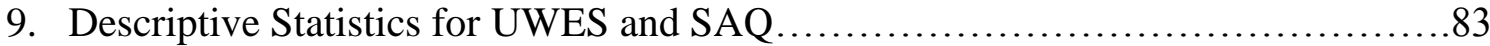

10. Bivariate Associations between Culture of Safety, OR Nurse Work Engagement, and

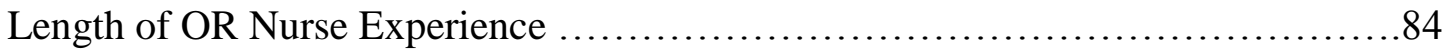

11. Associations between OR Culture of Safety, Length of OR Nurse Experience, and OR

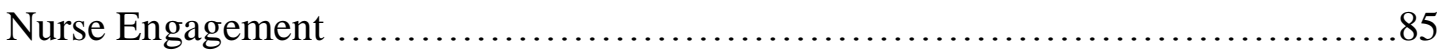

12. Summary of Predictors of OR Culture of Safety.............................. 85

13. Joint Display to represent Linked Results for the Exploratory Sequential Design. 


\section{LIST OF FIGURES}

FIGURE

PAGE

1. Barriers to adhering to safety standards/Reasons for normalization of deviance as described by OR nurses.......................................... 45

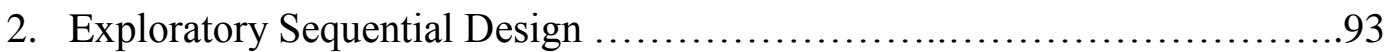




\section{CHAPTER I \\ INTRODUCTION}

The purpose of this dissertation was to present a mixed methods, exploratory sequential design study that explored safety-related practices in the Operating Room (OR) with a particular focus on normalization of deviance, and to examine factors that predict culture of safety in the OR, specifically, length of OR nurse experience and OR nurse work engagement. An introduction, three manuscripts, one of which provides an indepth exploration of the concept of normalization of deviance, and a conclusion chapter, which synthesizes the findings, comprise the dissertation.

Patient safety is a foundation of providing healthcare and has been compared to the provision of biological, physiological, and safety needs from the Maslow's Hierarchy of Needs model (Maslow, 1954; Ulrich \& Kear, 2014). Therefore, prioritizing the provision of patient safety within healthcare deserves sustained attention. However, there has been evidence that healthcare has fallen short of its responsibilities in this arena. For example, according to the Institute of Medicine's (IOM) 1999 report To Err Is Human: Building a Safer Health System, adverse events occurred in up to 3.7\% of annual admissions, and $13.6 \%$ of these events led to patient mortality. The 1999 IOM report stated that errors within the healthcare industry were responsible for approximately 44,000 to 98,000 patient deaths in the United States annually (Kohn, Corrigan, \& Donaldson, 2000). The report was an impetus that prompted healthcare systems to examine their personnel's safety-related behavior (King, 2010; Kohn et al., 2000). As a 
result of the concerning report, many healthcare facilities made strides to improve safety behaviors. For example, efforts were made to improve teamwork and to introduce transparent reporting of errors in a non-punitive manner (Espin, Lingard, Baker, \& Regehr, 2006). Despite significant endeavors to improve safety behaviors in healthcare organizations, medical errors persist, generating substantial personal and monetary cost (IOM, 2006). Vincent and Amalberti (2015) asserted that despite continued efforts to fortify safety, challenges persist, mainly because safety in healthcare tends to be like a "moving target" (p. 539) due to continued medical innovations that change the shape of how care is provided.

Safety in the operating room (OR) holds specific salience because it is one of the most complicated, information-intensive environments within healthcare (Christian et al., 2006; Wright, 2016; Link, 2019), and adverse events in the OR remain a substantial cause of mortality for surgical patients (Goldenberg \& Elterman, 2019). It is therefore imperative to examine patterns of performance and factors that affect safety in the OR.

Valuable insights on safety from other industries has been gleaned. For example, researchers who examined the causes of global catastrophes, such as the Chernobyl nuclear disaster, and the National Aeronautics and Space Administration's (NASA's) Challenger and Columbia Space Shuttle disasters, concluded that incidents like these are typically the result of many people breaking safety-related rules, a practice that appears innocuous on the surface. After an exhaustive exploration of the NASA disasters, Vaughan (1996) coined the term "normalization of deviance." The term placed a label on the phenomenon of gradually reducing safety standards to a new normal after a period of absence from negative outcomes. She concluded that the absence of negative outcomes 
tended to reinforce the behaviors associated with cutting corners, bypassing safety checklists, and ignoring alarms (Vaughan, 1996). This practice is specifically concerning in the OR arena as patient safety is heavily dependent on perioperative teams who adhere to safety standards.

Chapter two of the dissertation presents a concept analysis on normalization of deviance. Walker and Avant's (2019) concept analysis procedure was applied to organize the framework. The steps include the introduction of the concept, presenting the purposes of the analysis, outlining the uses of the concept, discussing the defining attributes, presenting cases related to the concept that include, a model case, a related case, and contrary case, identifying antecedents and consequences, and defining empirical referents (Walker and Avant, 2019).

Chapter three outlines the qualitative study that explored safety-related practices in the OR. The specific aims of the study included to: identify the primary safety concerns of OR nurses; explore the concept of normalization of deviance in the OR; and examine barriers and facilitators to the adherence to safety standards. The participants for the study included 10 OR nurses from the Louisville, Kentucky metropolitan area. The primary safety concerns that OR nurses reported were as follows: productivity pressures and inadequate staff to support workload. The qualitative interviews provided rich data, which illustrated the presence of normalization of deviance in the OR. Five themes emerged from the data that pointed to barriers to the adherence to safety standards and reasons for normalization of deviance in the OR, which included productivity pressures, generalized complacency, complacency related to length of experience, social pressures, and negative acculturation. Facilitators to the adherence to safety standards and protective 
factors against normalization of deviance included OR nurse engagement and supportive managerial relationships with OR nurses.

While examining barriers and facilitators to the adherence to safety standards, there was a particular focus placed on the length of OR nurse experience and OR nurse engagement. Length of experience and engagement and their association with safety are illustrated in Benner's (1984) Novice to Expert model, which explains how clinical experience leads to knowledge, engagement, and sound decision-making consistent with safety. Furthermore, it has been touted that the length of time that nurses remain in their roles is valuable because the knowledge and experience that is acquired cumulatively is a key component to quality care and overall safety for patients (Benner, 1984; Riley, Dearmon, Mestas, \& Buckner, 2016). In contrast, however to previous studies associating length of experience with safety, the concept of normalization of deviance suggests that deviations from standards occur over time, which is an indication that length of experience may be an encumbrance to safety practices.

The qualitative study findings demonstrated that while OR nurse experience can be linked to complacency, which can be antecedent to normalization of deviance, OR nurse engagement was touted to be a facilitator to the adherence to safety standards and a protective factor against normalization of deviance. In addition, supportive managerial relationships with OR nurses was deemed as a facilitator to the adherence to safety standards.

Chapter four outlines the quantitative study that aimed to examine the strength and association of relationships between the length of OR nurse experience, OR nurse engagement, and OR culture of safety. This chapter presents an overview on the culture 
of safety, which is reflected by the internalized values and behaviors of employees (Singer, Lin, Falwell, Gaba, \& Baker, 2009). A culture of safety is critical to thwart errors in the OR, and therefore, it is imperative to examine the factors that support a culture of safety and those that may attenuate it. The findings of the study indicated that OR nurse engagement had a strong, statistically significant relationship with OR culture of safety, and it was a significant predictor of OR culture of safety. In contrast, the relationship between OR nurse length of experience and OR culture of safety was not statistically significant.

Chapter five provides a synthesis of findings from both chapters three and four to present the mixed-methods, exploratory sequential design of the research. This chapter illustrates how two methods of research optimize the knowledge acquisition in the field of OR patient safety. For example, while social sciences have typically measured culture of safety by examining individual attitudes towards safety, other sciences, such as engineering, have traditionally assessed safety with a focus on the examination of systems (Pidgeon, 1998). Therefore, the combination of more than one approach to exploring OR safety was apt, whereby specific safety practices and systematic influences were explored within the tapestry of safety culture by using a mixed-methods, exploratory sequential design. Chapter five illustrates how the synthesized study results adds to the body of nursing knowledge, and implications for further research, practice implications, and policy were described.

In summary, OR nurses have patient safety concerns, normalization of deviance exists in the OR, and reasons for its occurrence include productivity pressure, generalized complacency, complacency related to length of experience, social pressures, and 
negative acculturation. Facilitators to the adherence to safety standards included OR nurse engagement and supportive managerial relationships with OR nurses.

It is crucial to disseminate the finding of this mixed-methods study so that normalization of deviance can be acknowledged as a prominent threat to OR patient safety. It is also essential to uncover the reasons why this occurs in ORs so that the problems existing in ORs can be addressed and mitigated. The results highlight the pertinence of OR nurse engagement and its role in mitigating risk in the OR. It is therefore critical to identify strategies to foster and sustain engagement in OR nurses. Finally, as evidence showed that supportive managerial relationships with OR nurses is a facilitator to the adherence to safety standards, efforts should be made to foster transparency and healthy, supportive communication between OR managers and OR nurses. 


\section{CHAPTER II}

\section{CONCEPT ANALYSIS: NORMALIZATION OF DEVIANCE}

\section{Introduction and Significance}

The term normalization of deviance was coined by Dr. Diane Vaughan, a prominent sociologist, who defined it as a phenomenon in which individuals and teams deviate from what is known to be an acceptable performance standard until the adopted way of practice becomes the new norm (Vaughan, 1996). The concept gained traction after Vaughan did an exhaustive exploration of the relationship structures and incidents within the National Aeronautics and Space Administration (NASA) that led to the disasters associated with both the Challenger and the Columbia space shuttles. For example, on January 28, 1986, the space shuttle Challenger disintegrated 73 seconds following the launch, which killed all seven crewmembers. The path of the shuttle's demise began after an O-ring seal in its right solid rocket booster failed at the shuttle launch. Its failure led to a rupture in the joint, which allowed pressurized burning gas from within the motor to dissipate. The cascade of events caused the separation and structural collapse of the external tank, essentially concluding with the shuttle blowing apart in a catastrophic manner. The relationship between normalization of deviance and the disaster lies within the realization that while the disaster itself was unanticipated, the problem that initiated the tragic cascade was not unknown (Gerstle, 2018).

Vaughan (1996) identified the phenomenon of gradually reducing safety standards to a new normal after a period of absence from negative outcomes, suggesting 
that the absence of negative outcomes tended to reinforce the behaviors associated with cutting corners, bypassing safety checklists, and ignoring alarms. In her book, The Challenger Launch Decision: Risky Technology, Culture, and Deviance at NASA, Vaughan specifically investigated the behavior of personnel leading up to the Challenger Space Shuttle disaster in 1986, and she illustrated how NASA personnel gradually tolerated anomalies and structural abnormalities within spacecraft joints. One of the primary contributors to the sense of comfort with danger was that there had been successful flights even when spacecraft joints were not sealing as they had been intended, which lulled NASA personnel into a false sense of security.

The concept aligns within Rasmussen's (1997) theory of migration to boundaries, which illustrated how front-line employees attempt to carry out work-related tasks with efficiency and productivity. Rasmussen explained that pressures on employees encourage adaptation to the environment, which may mean migrating to the boundaries of what is considered to be safe practice.

A prolific interest in organizational safety was generated in the 1990s, which was in part due to a culmination of well-publicized global disasters that occurred in the late 1980s, such as NASA's Challenger explosion, the Chernobyl nuclear disaster, the Exxon Valdez oil spill, and the London King's Cross Underground fire (Pidgeon, 1998). The exploration of global disasters has led researchers to conclude that system failures arise from a significant period of inattention to detail and a normalization of deviance (Gerstein \& Ellsberg, 2008; Pidgeon, 1998; Vaughan, 1996). Furthermore, it has been gleaned that disasters are typically the result of many people breaking safety-related rules, a practice that appears banal on the surface (Gerstein \& Ellsberg, 2008). 
Although the origins of the normalization of deviance concept arose within investigations into the safety practices of NASA (Vaughan, 1996), it has been determined that it has a ubiquitous presence in healthcare (Banja, 2010; Harvey \& Sotardi, 2017; Gerstle, 2018). In healthcare, deviation from sound safety practices may offer perceived advantages, such as efficiency from a time standpoint (Reid, 2014; Harvey \& Sotardi, 2017); some healthcare personnel speciously accept that deviations offer a path to increased productivity. Unfortunately, over time, short cuts are prone to becoming the cultural norm (Gerstle, 2018). This phenomenon unhinges the safety culture within an organization by gradually tolerating lower safety standards, which increases organizations' vulnerability to errors and adverse patient outcomes (Prielipp, Magro, Morell, \& Brull, 2010; Harvey \& Sotardi, 2017).

Normalization of deviance is particularly concerning in the operating room (OR) because, it is a complex, information-intensive, multi-faceted, fast-paced environment that has been described as hazardous for patients (Link, 2019) and deviating from safety standards can greatly increase surgical patients' risk for adverse events. According to Goldenberg and Elterman (2019), adverse events in the OR continue to remain a prevalent cause of mortality.

\section{Purpose}

The purpose of the analysis was to explore the concept of normalization of deviance, and the role it plays within the behavior of personnel in high-risk, healthcare environments, such as the OR. It is important to identify, expose, and acknowledge the phenomenon within high-risk healthcare environments. Gaining a better understanding of 
the concept opens doors to learning how to thwart its harmful consequences (Harvey \& Sotardi, 2017).

Analyzing the concept is also helpful to realize that there is something much more powerful at play than people who make mistakes in the workplace. A close analysis of examples where normalization of deviance occurred showed that people generally do not have intentions to be risky (Banja, 2010); rather, they are in situations where organizational pressures, such as time commitments and productivity pressures, push personnel to migrate past the boundaries of what is deemed to be safe (Amalberti et al, 2006; Price \& Williams, 2018). It is important for healthcare organizations to be cognizant of the phenomenon of normalization of deviance so that front-line personnel and leaders can be observant, intervene where there are indications that it is happening, and seek ways to impede its progression within organizations.

Although normalization of deviance exists in healthcare (Amalberti et al, 2006; Price \& Williams, 2018; Harvey \& Sotardi, 2017), there is a dearth of literature relating to its exact nature. One of the reasons cited is that while healthcare is submerged in rules and regulations, there is typically extra space for clinical judgement, and so it is difficult to define when a violation has actually occurred. In addition, due to the ubiquitous guidelines and recommendations, rigid adherence is uncommon, and these guidelines and standards are perceived more as recommendations than strict standards (Harvey \& Sotardi, 2017). Amalberti et al. (2006) averred that despite deviations being a serious threat to patient safety, the deviations are inadequately studied, and they concluded that the absence of adequate data should not undermine the problem. The danger of not exposing this phenomenon is that patient adverse events are likely to result when the 
breaching of safety standards go under-acknowledged over an extended period (Banja, 2010; Price \& Williams, 2018; Amalberti, et al., 2006; Harvey \& Sotardi, 2017).

\section{Uses of the concept 'Normalization of Deviance'}

Understanding the concept of normalization of deviance within high-risk

industries and acknowledging why personnel may deviate from safety standards illustrates how the stage may be set for major calamities to occur. According to Vaughn (1997), the relationship between policy decisions and errors is a prominent issue in all organizations, but in particular within organizations where there is potential for loss of life. Vaughan (1997) reported that most organizations have strategies to reduce errors by focusing on the immediate situation in which the error occurred as well as the people immediately involved, and much less attention has traditionally been given to the organizational system and its environment. Lessons learned from the NASA disasters can help people in other high-risk organizations, such as healthcare, focus on safety practices.

Patient safety has been a salient topic of conversation and literature for most of the past two decades. The 1999 IOM report, To Err is Human: Building a Safer Health System reported that as many as 98,000 patients die annually due to medical errors, which costs between 17 to 29 billion dollars (Institute of Medicine Committee on Quality of Health Care in, 2000). The sobering report was an impetus that has prompted healthcare systems to examine its personnel's safety-related behavior (Institute of Medicine Committee on Quality of Health Care in, 2000; King, 2010). The IOM report has also triggered healthcare systems to examine cultures of safety overall as well as how personnel drift from safety practices. The OR, in particular, is a high-risk environment where deviation from safety standards may lead to serious adverse patient outcomes. 
Although OR safety has been highlighted in the literature, and consequently many safetyrelated policies have been embraced, safety violations in the OR environment continue to be a problem (Zahiri et al., 2011; Goldenberg \& Elterman, 2019 ).

The incidence of unintentional retained foreign objects are estimated to occur in 1 in every 5,500 surgeries, and the outcomes for patients include reoperation, prolonged hospital stays, infection, obstruction, visceral perforation, and death (Steelman, Shaw, Shine, \& Hardy-Fairbanks, 2019). Mehtsun et al. (2013) reported that based on available malpractice data, US ORs had close to 10,000 never-events in the period between 1990 and 2010, and $6.6 \%$ of these events resulted in patient death. In addition, there was over $\$ 1.3$ billion paid toward malpractice claims related to those events. The researchers averred that these payments did not encompass the financial strain of legal fees, patient disability, lost work time, and defamation of the surgical team and facility involved. However, beyond the steep financial implications, working on solutions to prevent never events is required to prevent injury to patients. The researchers concluded that the number of never-events was probably much higher that what was reported in the study due to the numerous never-events that do not result in malpractice claims as well as the limit on reporting systems to capture each event (Mehtsun et al., 2013).

The Joint Commission required the use of the Universal Protocol in 2004, a process that mandated a carefully plotted process of patient and procedure identifications prior to surgery, the purpose of which was to increase patient safety standards by avoiding the occurrence of wrong site or wrong patient procedures. However, despite the best intentions, this adopted protocol did not meet its expected intentions; the standardized protocol has failed to prevent such events. Examination of the Universal 
Protocol's impediments concluded that general deviations from the intended protocol was a primary cause (Stahel, Mehler, Clarke, \& Varnell, 2009). In addition, King (2010) reported that deviant behavior of personnel was a substantial cause of errors in the OR. It is therefore critical to highlight the existence of normalization of deviance in the OR so that direct-care personnel and managers can acknowledge the phenomenon to assuage its progress and mitigate its consequences.

\section{Defining the Attributes of Normalization of Deviance}

According to Walker and Avant (2019), the defining attributes of a concept that are most commonly related the concept. A primary feature that is associated with normalization of deviance is productivity pressure (Price \& Williams, 2018; Harvey \& Sotardi, 2017). Productivity pressure may be defined as an increased emphasis on the quantity of services provided over the quality in the interest of the generation of revenue

(Wilbanks et al., 2018). This pressure typically stems from managerial personnel and creates a barrier to the adherence to safety standards that may be time-consuming (Harvey \& Sotardi, 2017).

Healthcare personnel's deviant behavior from safety practices does not stem from malicious intent (Banja, 2010; Harvey \& Sotardi, 2017); deviant behavior is typically the result of a perceived need for rational and necessary steps to increase productivity. Due to productivity pressures that are applied to personnel in the work environment, rationalization of deviant behavior becomes perceived as legitimate and necessary (Banja, 2010; Harvey \& Sotardi, 2017). Banja (2010) alluded that normalization of deviance can occur when administrators deliver strict rules and regulations to direct-care employees, while concurrently demanding a high level of productivity. The application of 
this kind of pressure encourages front-line workers to take short cuts and use workarounds to get a task accomplished without all of the steps that some regulations demand. Some argue that complete adherence with all of the rules would never make it possible to achieve the productivity that is demanded of them (Banja, 2010). Productivity pressure is an example of an unseen complexity within an organization that make it difficult for personnel to always adhere to safety protocols (Reason, 1990), and therefore it is a barrier to safety.

Productivity pressure is a dominant facet of working within the OR, specifically because ORs are primary generators of revenue for hospitals (Peters, White, \& Mahalvan Brenk, 2013; Rothstein \& Raval, 2018). In the OR, deviations are supportive of short-term efficiencies and help perioperative personnel to maintain a busy schedule with the productivity pressures that are placed on them. The threat to patients becomes more apparent when violations are accepted over time and when personnel become blind to the associated risk. The concept is congruent with Rasmussen's (1997) theory of migration to boundaries, which suggested that direct-care employees are pressured to work with distinct focus on efficiencies and productivity, and in an attempt to honor productivity, safety boundaries becomes blurred.

\section{Model 'Normalization of Deviance' Case}

Vaughan (1996) presented the classic case of normalization of deviance in her book The Challenger Launch Decision. Vaughan provided a deep analysis of how NASA's space shuttle Challenger disaster occurred in 1986. According to Vaughan (1996), nine shuttles were launched successfully in 1985, the year prior to the Challenger disaster. Seven of these shuttles showed signs of erosion; two had damage beyond 
anything NASA had seen before. After each of these flights, engineers at NASA raised concerns about O-ring erosion, which is deterioration of the rings designed to be situated between two or more parts during assembly of the shuttle to create a seal at the joining interfaces. Despite memos, presentations, and acknowledgement about the O-ring concerns, NASA flights continued as usual. Vaughan concluded that the concept of normalization of deviance explained how NASA initially accepted O-ring anomalies; NASA personnel succumbed to lower standards, and flying shuttles with damaged Orings became the new norm (Vaughan, 1996). By 1985, there had been evidence of Oring erosion in seven of the nine shuttle launches. The engineers who oversaw the launches recognized that there was potential for calamity, particularly during a launch months earlier when extensive O-ring damage was identified during a post-flight review. The January 1986 launch on that fateful day however was planned despite existing problems. The personnel were willing to take the risk because previous flights that had been deemed successful guided the perception that there were no inherent danger (Gerstle, 2018).

Dr. Vaughan was also involved in the investigation of NASA's Columbia disaster in February 2003, which was 17 years after the Challenger disaster, and discovered a similar sequence of events. The cause of this tragedy was a foam debris strike, which evidently had been occurring on shuttle flights prior to the disaster. As with the 1986 incident, NASA personnel had again succumbed to normalization of deviance, during which they had been aware of foam debris strikes and had tolerated lower safety standards because they perceived the risk to be acceptable (Vaughan, 2006). 
In the 2008 publication of Flirting with Disaster; Why Accidents are Rarely

Accidental, Gerstein and Ellsberg concurred with Vaughan (1996) that normalization of deviance played a primary role in NASA's disaster. The authors stated that knowingly accepting such high risk in this way is similar to Russian Roulette, an activity which carries enormous peril; they concluded that no matter how many times Russian Roulette is played, it is still never safe. Instead, however, NASA personnel increased their comfort level and confidence by focusing on previous successful ventures and had forgotten to be fearful. According to Gerstein and Ellsberg (2008), a better approach would have been to assess each occurrence of O-ring erosion as perilous and to demand a rigorous resolution to problems as they occurred. Undoubtedly, the extra time allocated into remedying anomalies would result in delays and cost, but with an added benefit of high reliability (Gerstein \& Ellsberg, 2008) .

Gerstein and Ellsberg (2008) also articulated the details of the Ukrainian Chernobyl nuclear disaster in 1986 when radioactivity 90 times greater than the Nagasaki and Hiroshima bombs was discharged into the atmosphere surrounding the nuclear plant site, and concentrated toxic and carcinogenic plume spewed into the densely populated region surrounding the Chernobyl nuclear plant site in northern Ukraine. According to Gerstein and Ellsberg (2008), there were well-known signs of deviant safety practices preceding the event; however, personnel chose to under-report and under-acknowledge short cuts. Essentially, the deviant practice had become normalized over time, and disaster struck (Gerstein \& Ellsberg, 2008).

The lessons learned from global disasters can be aptly applied to healthcare because while there are not shuttles and nuclear power plants to manage in healthcare, 
there are certain parallels in relation to managing risk (Gerstle, 2018). Banja (2010) concurred with Reason (1990) who stated that disaster in healthcare is almost certain when omissions remain unresolved or under-acknowledged for an extended period. Therefore, what may begin as a perceived innocuous deviation from a safety practice will somehow become "normalized" over time due to the repetition of the deviation (Vaughan, 1996; Gerstle, 2018). Unfortunately, these deviations become engrained in the culture of the organization, which is a serious cause of the unhinging of a safety culture.

\section{Borderline, related, and contrary cases}

Odom-Forren (2011) examined how normalization of deviance existed within perianesthesia nursing. During her investigation, colleagues gave examples of when safety standards had been abandoned, or deviations from safety practices occurred. Although many of the responses that she received were standard cases of normalization of deviance, some responses alluded to borderline cases of the normalization of deviance. These examples illustrated how adverse patient events could occur due to violations in safety protocols and standards. For example, an RN reported that she was admonished in her workplace because she would not administer fentanyl from an unlabeled syringe. Other colleagues reported that they were rushed in the preoperative arena and admitted that individualized patient care suffered as a result. A common theme was "we don't have time for that" (Odom-Forren, 2011) in relation to adhering wholly to safety protocols that may have been perceived as inefficient or unnecessary. Odom-Forren (2011) described how each $\mathrm{RN}$ in the examples did not perceive that this one action would lead to any kind of harm for patients. However, she pointed out that as violations occur, nurses become desensitized to the potential implications, and it becomes easier to normalize substandard 
ways of performing tasks while renouncing some key safety practice measures in its wake.

A related case of normalization of deviance was found in an article studying the occurrence of patient harm and death due to the mix-up of enteral feeding systems and intravenous (IV) systems. Simmons et al. (2011) reported incidences that included accidental connections of tubes with feedings that were intended for enteral use to an IV system. The authors concluded that the accidental misconnections were primarily related to a "universal" connector that can be used for both systems (Simmons, Symes, Guenter, \& Graves, 2011). Based on the case reviews, a redesign of the connectors was recommended so that enteral feeding connectors would only be compatible with enteral feeding, and IV connectors would only be compatible with IV systems to minimize or prevent mistakes. However, despite documented evidence that these connectors cause patient harm and death, there had been an unwillingness to change, and progress was sluggish to remedy the danger. Decisions relating to the tubing were made at an administrative level with a focus on productivity and without a clear sense of patient safety, and the universal connector production continued and were distributed for use (Simmons et al., 2011).

Gerstein and Ellsberg (2008) illustrated an example of a related case of normalization of deviance when they reported details on a highly publicized disaster that involved the one-time popular prescription medication Vioxx manufactured by the pharmaceutical giant Merck \& Company. During the period between 1999 and 2004, this medication resulted in over 100,000 injuries or deaths, and although there were known risks associated with the medication but it remained on the market because of exorbitant 
profits for the pharmaceutical company (Gerstein \& Ellsberg, 2008).

An example of a case contrary to the normalization of deviance would include a situation where OR nurses take excessive measures to apply safety standards to their patients without a rational evidenced-based practice approach. In addition, actions would be taken without any regard for time, cost, and efficiencies. This example would present a precarious platform on which safety measures would be provided to patients but it would be an unsustainable approach to care delivery.

\section{Antecedents and Consequences to Normalization of Deviance}

According to Walker and Avant (2019), an antecedent must be in place before the occurrence of the concept and is not the same as a defining attribute. A primary antecedent to normalization of deviance is a generalized complacency related to the absence of adverse events (Vaughan, 1996; Harvey \& Sotardi, 2017; Price \& Williams, 2018). Vaughan (1996) suggested that the absence of negative outcomes leads personnel to perceive that adverse events will not happen, which tends to fortify behaviors associated with taking shortcuts and bypassing safety protocols in their entirety. Furthermore, there tends to be an insensitivity to a deviant practice whereby it no longer feels wrong (Vaughan, 1996; Price \& Williams, 2018).

There is also a sense of complacency when rules and standards are perceived as irrational and inefficient (Banja, 2010; Odom-Forren, 2011). Banja (2010) illustrated an example of a protocol aimed to prevent narcotic diversion that requires nurses to enter their passwords as an indication that they have witnessed the safe waste of an incomplete administration of the contents of a narcotic vial. However, as nurses are frequently rushed because of competing demands in the workplace, they may perceive that the task of 
asking a colleague to enter a password in the automated medication dispenser as an inconvenience that hampers efficiency, especially in light of the relative infrequency of narcotic diversion. Thus, in an effort to increase efficiency, the may nurses share passwords so that they can enter each other's passwords in the event that the automated system requires a second password. The nurses in this situation are reacting to what they see to be an inefficient use of their time, and they fail to see that the perceived avenue of efficiency widens the gap of vulnerability for OR nurses to misuse narcotics. Therefore, the deviant practice negates any benefits from the intended aim to avert narcotic diversion (Banja, 2010).

The consequences of normalization of deviance and its association with disaster has been well documented (Banja, 2010; Gerstein \& Ellsberg, 2008; Vaughan, 1996). This concept is a common pitfall for all personnel within high-risk organizations. Collins et al. (2014) concluded that accidents are seldom the consequence of isolated errors made by individuals; rather, accidents occur as a result of many errors occurring within a flawed system. A common remedy for mistakes in the workplace consists of reprimand of the person who made the error, and the reemphasis of the rules or policies. Reid (2014) argued that many of the traditional approaches to addressing workplace errors are ineffective and asked rhetorically how can reasonable people be expected to stop carrying out their specific ways of practice if there are specific barriers to adhering to safety standards.

Collins et al. (2014) referred to the Swiss Cheese model from Reason (2000) as a metaphor to illustrate how organizations without several layers of defense are vulnerable to catastrophe. Reason (2000) suggested that humans tend to deviate and make errors, but 
catastrophes can be avoided if organizational systems are layered with enough defenses to recognize deviations early and make modifications to thwart major ill effects. Price and Williams (2018) also applied the Swiss Cheese model in their explanation of how adverse patient events can occur as a consequence to repeated deviations over a period, and reported that that adverse events occur when several critical factors align, which represent the holes in Reason's Swiss Cheese model.

\section{Empirical referents}

The last step in a concept analysis is the identification of empirical referents, which are categories that illustrate the occurrence of the concept (Walker \& Avant, 2019). While there is currently not an instrument to measure normalization of deviance, occurrences can be seen in practice or captured qualitatively.

Deviations from safety protocols are visible in the workplace under many guises, and each carry various amounts of risk. McNamara (2011) presented reflections of normalization of deviance in the OR as she discussed how OR personnel deviate from well-known guidelines set in place by the AORN. For example, the standards to prevent surgical site infection are typically well known by OR personnel, yet deviations from the standards may be seen. Examples include: personnel may gown off the back table instead of gowning off a separate flat surface so that the sterile back table is not contaminated by the risk of touching it with non-sterile, ungloved hands; personnel may remove gloves and retrieve clean supplies without washing their hands; personnel may not supervise opened sterile supplies in an OR; or personnel may move a sterile table by grasping its edges below the level of the sterile field after they have scrubbed (McNamara, 2011). Other common areas of deviation include reusing a "single-use" device, failure to follow 
each step of safety checklists, and inadequate training to complete a task (Reid, 2010).

While each deviation may be perceived to be benign and innocuous, the cumulative effect of deviations reduce safety standards. OR personnel, have mistakenly accepted that negative events, such as surgical site infection, wrong-site surgery, or retained surgical items are the consequence of one person committing a single act of negligence. However, history has taught that the occurrence of adverse events typically are reflective of multiple people deviating from safety protocols over a period (Banja, 2010). The tolerated mediocrity can set the stage for a perfect storm, where patients can be our casualties (Reid, 2014; Price \& Williams, 2018).

\section{Summary}

Normalization of deviance has been described as the acceptance of lower standards due to a gradual acceptance of deviant behavior by personnel. NASA decided to fly space shuttles with what they deemed as acceptable risk, but which resulted in disaster on more than one occasion. Lessons learned from NASA and other global disasters are salient reminders of what can happen in healthcare, particularly in the OR, if personnel within the field deviate from safety standards.

Vaughan (1996) averred that most disasters have had an "incubation period" of deviation that occur without acknowledgement. When deviations accumulate, overtime the culture is modified to accept these deviations as cultural norms. She also clarified that repetition of what seems to be trivial, and the banality of daily routine, disguises a cumulative danger that may only be fully acknowledged in hindsight.

Deviation from rules become normalized if people who see them fail to intervene (Banja, 2010). In some situations, personnel may not be assertive enough to speak up or 
they may feel powerless within an organization to intervene. Safety experts within the healthcare industry stated that undetected and underreported errors thwart the recognition of threats to patient safety and impede the progress on learning how to assess and avoid errors (Institute of Medicine Committee on Quality of Health Care, 2000). Gerstein (2008) referred to a term "politics triumphing over safety," where supervisors may not disclose some unsafe practices within a department to upper management in an attempt to look better to superiors.

Because the normalization of deviance is inimical to high quality care and safety, oversight and monitoring should be ongoing within healthcare facilities. The optimal time to intervene in the normalization of deviance is as soon as it is recognized because allowing unsafe behavior to continue further corrodes an organization's defense system (Banja, 2010). Lessons learned from catastrophes in the past have shown that normalization of deviance may be present for extended periods before any catastrophe emerges; however, the longer it happens, the more likely it is that catastrophe will uncover itself (Vaughan, 1996).

System administrators must acknowledge that organizations have potential for normalization of deviance. When patient adverse events occur due to the normalization of deviance, system administrators need to recognize the intricacies of the cause of the error, rather than placing the blame on the person on the front line of the error. Deviance is a reflection of a flawed safety system that needs to mended (Price \& Williams, 2015). It is imperative that the reasons for this phenomenon are explored within organizations so that it can be discussed openly and become transparent. Support should be given to direct-care nurses so that barriers can be removed to adhere to intended safety protocols. For 
example, unrealistic time pressures should not be placed on front-line employees, staffing ratios should be observed so that there are enough personnel to complete tasks safely and efficiently, and rewards should be given for safety as opposed for productivity. 


\section{CHAPTER III EXPLORING SAFETY-RELATED PRACTICES IN THE OPERATING ROOM (OR) Background}

Normalization of deviance is a phenomenon in which individuals and teams deviate from what is known to be an acceptable performance standard until the adopted way of practice becomes the new norm. In healthcare, deviation from sound safety practices may offer perceived advantages; some healthcare personnel fallaciously believe that deviations offer a path to increased productivity. Over time, however, this kind of deviance is susceptible to becoming a cultural norm. This phenomenon erodes the safety culture within an organization by gradually accepting lower safety standards, which increases organizations' exposure to risk. It can be particularly concerning in complicated, high-risk work environments, such as the operating room (OR) arena.

\section{Method}

This qualitative study used a focused ethnography design in which a purposeful sample of OR nurses with maximum variation was interviewed.

\section{Purpose}

The purpose of this study was to: 1) identify the main safety concerns in the OR from the perspective of practicing OR registered nurses; 2) explore the concept of normalization of deviance in the OR; and 3) identify perceived barriers and facilitators to adhering to safety standards. 


\section{Findings}

Specific examples of normalization of deviance in the OR included: productivity pressures, generalized complacency, complacency related to length of experience, social pressures, and negative acculturation. Facilitators to adhering to safety protocols included nurse engagement.

\section{Introduction}

Normalization of deviance is a phenomenon in which people and teams deviate from what are accepted as standard performance measures until the adopted way of performance becomes a new and accepted norm (Vaughan, 1996). The phenomenon may be pervasive within organizations because people within an organization become so insensitive to deviant practice that this type of practice is no longer perceived as "wrong." Insensitivity to deviance typically occurs perfidiously and gradually because adverse events do not generally occur until there is an alignment of other critical factors (Price \& Williams, 2018).

The concept lies within Rasmussen's (1997) theory of migration to boundaries, which illustrates how front-line employees attempt to carry out work-related tasks with efficiency and productivity. Rasmussen (1997) explained that pressures on employees encourage conformation to the environment, which may mean drifting to the boundaries of what is known to be safe practice. When the drift becomes more frequent and accepted, it has potential to project outside the boundary of safe practice (Rasmussen, 1997). Eventually this behavior may lead to adverse outcomes (Amalberti, Vincent, Auroy, \& de Saint Maurice, 2006; Harvey \& Sotardi, 2017; Price \& Williams, 2018). Research on global errors and calamities, such as the Chernobyl nuclear accident and 
National Aeronautical Space Administration's (NASA) Challenger and Columbia Space Shuttle disasters, has concluded that catastrophe is typically the result of many people breaking safety-related rules, the behavior of which appears innocuous on the surface (Gerstein \& Ellsberg, 2008). Vaughan (1996) identified the concept of normalization of deviance when she initially investigated the NASA Challenger disaster. Her extensive work related to both the NASA Challenger and Columbia disasters has been shared amongst other organizations, and it has helped industries, such as healthcare, recognize normalization of deviation and its uncertain consequences.

Although the origins of normalization of deviance concept arose within investigations into the safety practices of NASA (Vaughan, 1996), it has a pervasive presence within the healthcare industry (Banja, 2010; Harvey, 2017; Price \& Williams, 2018); however, there is ambiguity relating to its precise nature (Amalberti et al. 2006). There is a perception that deviation from safety practices in healthcare offers efficiency and increased productivity (Reid, 2014; Price \& Williams, 2018). Over time, however, deviations and short cuts are prone to normalization. Normalization of deviance unhinges the safety culture by gradually tolerating mediocrity, and this increases organizations' vulnerability to adverse events (Prielipp, Magro, Morell, \& Brull, 2010; Banja, 2010).

Normalization of deviance is particularly concerning in the Operating Room (OR) because, it is a complex, information-intensive, multi-faceted, fast-paced environment that has been described as hazardous for patients (Link, 2019). Deviating from standards of care can in this type of environment greatly increase surgical patients' risk for adverse events. According to Goldenberg and Elterman (2019), adverse events in the OR continue 
to remain a prevalent cause of mortality.

The study aims were to: 1) identify the main safety concerns in the OR from the perspective of practicing OR registered nurses; 2) explore the concept of normalization of deviance in the OR; and 3) identify perceived barriers and facilitators to adhering to safety standards.

\section{Background and Significance}

The Institute of Medicine's (IOM) 1999 report To Err Is Human: Building a Safer Health System, concluded that adverse events occur in up to $3.7 \%$ of annual admissions and $13.6 \%$ of these events lead to patient mortality. The report further estimated that error within the healthcare industry was responsible for approximately 44,000 to 98,000 patient deaths in the United States annually. The IOM concluded that errors within healthcare were not the result of incompetent people making mistakes; rather, the cause of healthcare errors is multi-faceted (Kohn, Corrigan, \& Donaldson, 2000).

This report was an impetus that prompted healthcare systems to examine their personnel's safety-related behavior (King, 2010; Kohn et al., 2000). As a result, many healthcare facilities made efforts to fortify safety-related processes, such as strengthening teamwork and introducing better reporting systems in a non-punitive manner (Espin, Lingard, Baker, \& Regehr, 2006). Despite significant efforts by many health care organizations to improve safety behaviors, medical errors persisted, generating substantial personal and monetary cost (IOM, 2006). Vincent and Amalberti (2015) averred that safety within the healthcare system is unique to safety in other organizations because as medical innovation continues, more types of patient adverse events are seen as preventable, therefore, patient safety margins get wider, making safety in healthcare a 
"moving target" (p. 539). In essence, there is a range of vulnerabilities within healthcare systems, and there are a myriad of contributors to errors. Therefore, ongoing efforts of identification and acknowledgement of patient adverse events (and antecedents to such) should continue to be a salient provocation in healthcare and play a role in guiding safety improvement strategies (Vincent, Carthey, Macrae, \& Amalberti, 2017).

Maintaining a safe environment for patients in the OR has specific relevance, particularly because the OR is an information-intensive environment with complicated technology managed by multi-disciplinary team members who carry out high-risk interventions (Jones \& Durbridge, 2016). Despite efforts to mitigate risk and maintain a safe environment for patients, adverse events in the OR remain a substantial threat to surgical patients (Zahiri et al., 2011; Goldenberg \& Elterman, 2019).

The incidence of unintentional retained foreign objects are estimated to occur in 1 in every 5,500 surgeries, and the outcomes for patients include reoperation, prolonged hospital stays, infection, obstruction, visceral perforation, and death (Steelman, Shaw, Shine, \& Hardy-Fairbanks, 2019). Between 1990 and 2010, US hospitals had close to 10,000 OR-related never-events, which are occurrences that include unintentional retained foreign objects, wrong-site/wrong-procedure/wrong-person surgeries; $6.6 \%$ of these never events resulted in patient death. (Mehtsun, Ibrahim, Diener-West, Pronovost, \& Makary, 2013).

In an effort to avoid the occurrence of wrong site or wrong patient procedures, the Joint Commission required the use of the Universal Protocol in 2004, a process that mandated a carefully plotted process of patient and procedure identifications prior to surgery (The Joint Commission, 2004). However, this adopted protocol did not meet its 
expected intentions, and failed to prevent such events (Stahel, Mehler, Clarke, \& Varnell, 2009), which was primarily due to lack of OR personnel compliance (Stahel et al., 2009; Banschbach, 2009). Impediments to the Universal Protocol's success included inconsistencies in site-marking procedures, discrepancies in education for staff, and general deviations from the intended protocol . King (2010) reported that deviation from safety protocols over a period that had become normalized was a primary reason OR for patient adverse events.

Exploration of examples of normalization of deviance showed that people generally do not intentionally engage in risky behavior (Banja, 2010; Price \& Williams, 2018). Rather, they are in situations where organizational pressures, such as time commitments and productivity pressures, push personnel to migrate past the boundaries of what is deemed to be safe (Rasmussen, 1997; Amalberti et al, 2006; Dekker, 2011; Price \&Williams, 2018). This factor holds particular relevance in the OR, which can be one of the most prominent sources of revenue to hospitals. The OR has been referred to as the "financial hub of any hospital" (Rothstein \& Raval, 2018, p 79), sometimes accounting for $65 \%$ of hospital margins (Peters, Young, White, \& Mahal-van Brenk, 2013). Therefore, OR personnel may be more prone to migrating past boundaries of safe practice due to productivity pressures driven by substantial revenue generation.

While historically, organizations generally focused on incident reports and investigative efforts to assess safety, there is wide acceptance that adding assessments that both anticipate safety risks and include human factors is needed in the future (Vincent, Carthey, Macrae \& Amalberti, 2017). Therefore, assessing the occurrence of normalization of deviance and the reasons for its occurrence is highly warranted, 
particularly in the OR. There has been a paucity of research exploring normalization of deviance in the OR.

\section{Pilot Study}

In December 2017, a pilot study conducted separately from this current study in was driven by a threefold purpose: 1) to examine the feasibility of the qualitative approach exploring safety concerns that OR nurses had; 2)to gain insight into the existence of normalization of deviance; and, 3) to explore reasons for its occurrence. The pilot study data, which was not include in this study, contributed helpful insights about re-structuring the study design as well as modifying the interview questions to better understand the barriers and facilitators regarding OR safety standard adherence (Wright, 2018).

Pilot study findings $(\mathrm{N}=10)$ confirmed the presence of normalization of deviance, and the exposed six themes underlying the occurrence of normalization of deviance included generalized complacency, productivity/time pressures, workplace incivility, infobesity, and variation in experience (Wright, 2018). This current study was based on the pilot data collection and analysis. As a result, the purpose of the current study was modified to include the examination of primary safety concerns of OR nurses, to explore examples of normalization of deviance, and to examine barriers and facilitators to the adherence to safety standards. Interview questions were amended to include the role of specific nurse characteristics, such as length of OR nurse experience, nurse engagement, and nursing's role in fortifying or assuaging safety-related practices (Wright, 2018). 


\section{Research Design and Methods}

This cross-sectional, qualitative study features a focused ethnographic design. Focused ethnography is a branch of traditional ethnography in which specific elements of a culture are examined against the backdrop of a larger more complex culture (Wolf, 2010). According to Richards and Morse (2013), ethnography provides the researcher with an opportunity to describe cultural norms, perspectives, characteristics, behaviors, and patterns. The focused ethnographic design is appropriate for the exploration of a subcultural group rather than a cultural group (Richards \& Morse, 2013). Thus, this design was apt to use to explore safety behaviors and normalization of deviance within the subculture of OR nurses.

\section{Sample}

Participants were purposely sampled based on their field-specific knowledge of OR nursing. Because OR nursing is a nursing sub-culture with its own rules, knowledge, skill-set, and behaviors, purposive sampling is an appropriate technique for a focused ethnography. Specifically, maximum variation sampling was applied, the goal of which was to include OR nurses who practice at various ORs with varying amounts of clinical experience so that multiple perspectives could be gathered. The OR nurse participants were recruited from the healthcare systems within the Louisville metropolitan area. Eligibility criteria included currently licensed registered nurses who work primarily in an OR setting, over the age of 18, and English-speaking.

\section{Recruitment}

A group recruitment email was sent to the members of the Kentuckiana Association of Operating Room Nurses (AORN) chapter, which serves OR nurses within 
the metropolitan areas of Louisville, Kentucky and the adjacent Southern Indiana area. The email informed the nurses about the study, its purposes, and the request for participants. The Kentuckiana AORN Chapter association has over 150 OR nurse members, many of whom are employed at various OR facilities within the Louisville metropolitan area. In addition, announcements were made at monthly OR staff meetings at a Louisville hospital. Flyers were also dispersed to Kentuckiana AORN members containing information about the study as well as the contact information of the researcher. OR nurses were given the opportunity to call or email the researcher with questions about the study or to ask the researcher about participating in the study. After contacting the researcher, OR nurses were screened for eligibility, were provided an explanation of the study purpose and process, and were provided assurance confidentiality. If the OR nurses showed interest in participating, arrangements were made to meet them individually in a private location where the interview could take place. A non-monetary incentive of a coffee mug, worth about $\$ 10.00$, was offered to OR nurses who agreed to participate.

\section{Procedure}

Participants for the study included OR nurses who were employed within the Louisville, Kentucky metropolitan area in a direct-care OR setting for at least 20 hours per week. Each participant signed an informed consent that included full disclosure of the study aims. The plan for maintaining confidentiality of data was explained. Data regarding the facilities in which the participants worked were not documented. One-onone interviews were performed at locations where a private conversation could be held and one that was convenient for the participants, such as the participant's home, the 
researcher's office at University of Louisville School of Nursing, or at a coffee shop. Interviews did not take place in the participants' work setting. Interviews were digitally audio recorded.

Five standard open-ended questions, some of which included additional probe questions (Table 2, Appendix), were employed to conduct the qualitative interviews. The questions did not focus on the occurrence of adverse events in the OR; rather the interview questions addressed daily practices that were concerning and practices that potentially set the stage for an adverse event to occur. Specific exploration occurred in relation to the nurses' perceptions of the length of clinical experience and nurse engagement as factors that affect safety practices. In addition, a demographic data form was completed by each participant. Data gathered through this form included: length of clinical experience measured in consecutive years, gender, age, level of education, status of OR nurse certification, such as Certified Nurse in the Operating Room (CNOR), and number of operating rooms within the department where OR nurses were employed.

\section{Data Management}

Trustworthiness was a key component to the rigor and credibility of the study; therefore, prolonged engagement for approximately one hour with each participant occurred to ensure that each participant had adequate time to relay his or her answers (Balkin \& Kleist, 2017). A digital recorder was used to record each interview. Analytic memos were written before, during, and after data collection. This process is consistent with Wolf's (2010) suggestion that researchers should reflect the participants' viewpoint by carefully selecting specific comments, phrases, and expressions that fit the purpose of the ethnographic study. The memos written before data collection documented any biases 
that the researcher may have possessed. Awareness of self, biases, and creed before and during data collection was crucial, specifically because the researcher has a history of OR nursing (Richards \& Morse, 2013). In addition, the phrase "normalization of deviance" was purposefully withheld from being part of any interview questions to avoid influencing the participants' words. The memos written during and after the data collection reflected concepts and patterns as they emerged.

Ten interviews were completed within a six-week period. To protect the participants' information, after each interview, the recorder was transported within a lock box, the code for which was only known by the researcher. The recorded interviews were downloaded to an encrypted, password-protected computer as soon as possible after which the original recording was deleted from the recording device. Data were transcribed by a professional transcriber, and the transcripts were checked for accuracy by the researcher.

\section{Data Analysis}

Demographic data were entered into SPSS Verison 25.0 (IBM Corp, 2017) to attain descriptive statistics related to the participants. Transcripts from the interviews were downloaded to the software Dedoose Version $\mathbf{8 . 0 . 3 5}$, which is web application for managing, analyzing, and presenting qualitative and mixed method research data (Dedoose, 2018). The data were explored by reading through the entire content, and initial memos were written to gather thoughts as they surfaced.

The analysis of the transcripts overall was an iterative process that involved disassembling the narrative text into compartments of information called codes (Creswell \& Plano Clark, 2018). Topic coding occurred initially and involved creating categories 
for the data as well as determining where the categories fit within the ideas of the researcher. The primary purpose of this was to identify material for description, categorization, or reflection. The topics were identified as each line of the transcript was read. This process is consistent with Wolf's (2010) suggestion that researchers should reflect the participants' viewpoint by carefully selecting specific comments, phrases, and expressions that fit the purpose of the ethnographic study. The story of the participants reflected their perspective, and details were recorded so that a larger context could be gleaned (Richards \& Morse, 2013).

Analytic coding occurred secondarily after topic coding, providing a different platform on which to allow new deeper categories, concepts, and themes to emerge (Richards \& Morse, 2013). This also presented an opportunity to compare themes that had emerged from the pilot study data analysis. In addition, comparisons and contrasts were drawn to form categorical clusters, which generated meaningful themes relative to the aims of the study. Interrelated themes were identified, and grouped coding was done (Creswell \& Plano Clark, 2018) when evidence emerged to suggest that themes overlapped in some contexts.

To increase rigor and trustworthiness, a peer debriefing process took place whereby an expert in the field checked the data for congruence (Balkin \& Kleist, 2017). Each description was confirmed by comparing it to the interview context, and the transcripts were reviewed for data that was disproving, discrepant, or negative (Richards \& Morse, 2013; Balkin \& Kleist, 2017). In addition, the researcher applied a member check process confirming interview details with two of the participants. Participant feedback assured that the collected data and coding of that data fit accordingly. An audit 
trail was maintained to reflect research events and decisions in such a way that an independent auditor could access and understand the process s (Balkin \& Kleist, 2017).

\section{Results}

The participants included ten registered nurses who were working at least 20 hours per week in an OR within the Louisville metropolitan area. The mean age of the participants was 43.4 years $(\mathrm{SD}=3.03)$ (Table 1). The mean length of overall nurse experience was 17.4 years (SD 11.45), and the mean length of experience specific to the OR was 11.1 years (SD 10.7). The mean number of operating rooms within the OR units where the participants were employed was 10.7 (SD 5.51). One of the participants had CNOR certification, and nine did not. Four of the participants held an Associate degree in nursing, and six participants held a Baccalaureate degree in nursing.

Table 1. Descriptive Characteristics of Participants $(\mathrm{N}=10)$

\begin{tabular}{lr}
\hline Characteristic & Mean (SD) \\
\hline Age in years & $43.4(3.03)$ \\
Length of experience as a nurse & $17.4(11.45)$ \\
Length of experience in the OR & $11.1(10.70)$ \\
Number of ORs in place of employment & $10.7(5.51)$
\end{tabular}

\section{CNOR}

Yes

No

$9(90)$

Education Level 
Diploma

Associates degree

Baccalaureate degree

Graduate degree
$0(0)$

$4(40)$

$6(60)$

$0(0)$

Note. $\mathrm{M}=$ mean; $\mathrm{SD}=$ standard deviation

\section{Aim 1) Primary Safety Concerns in the OR from the Perspective of Practicing OR RNs}

Primary safety concerns in the OR were categorized into two main themes:

productivity pressures and inadequate staff to support workload. Additional concerns that were reported included: "infobesity;" inconsistency of order entry; general complacency of staff. Examples of quotes supporting these themes and overall concerns are illustrated in Table 3 in the Appendix.

\section{OR Safety Concern- Theme 1: Productivity Pressures}

Productivity pressures may be defined as an increased emphasis on the quantity of services provided over the quality in the interest of revenue generation (Wilbanks et al., 2018). Eight out of ten participants suggested that productivity was a prevalent safety concern where they work. Examples of quotes that contribute to this theme include: "Efficiency has kind of given way to patient safety." and "We're constantly being made to cut corners for efficiency versus reviewing patient records, making sure that we have everything, that the patient is stable, all of our ducks lined up in a row." In addition, one participant highlighted that the preoperative assessment as well as the delivery of a 
complete patient hand-off report to the recovery room staff were inadequate, which was primarily due to the pressure of having a fast turnover time between surgeries.

\section{OR Safety Concern-Theme 2: Inadequate Staff to Support Workload}

An inadequate staff support system was touted as a safety concern, as evidenced in four out of ten participant statements. Examples of quotes that contribute to this theme include: "The top patient safety [concern] is patient positioning on a narrow bed and somebody falling off. At our facility, we don't have a whole lot of staff, so trying to bring the patient into the bed by yourself always concerns me." and "Room turnovers are tough because our staffing situation right now is not ideal as far as people that we hire to turn over rooms and stuff." Although the latter quote reflects a concern of inadequate staff support, there is an embedded reflection of productivity pressure because the participant is relating this concern with getting surgical OR suites cleaned and prepared between surgeries.

\section{Additional OR Safety Concerns}

OR participants also reported additional OR safety concerns that included "infobesity;" inconsistency of order entry; general complacency of staff. Although these topics were coded, they were not categorized as themes because they were not seeN as common threads that ran through the data (Richards \& Morse, 2013).

Infobesity, a relatively new term that emerged within the modern urban dictionary (Rogers, Puryear, \& Root, 2013), refers to the onslaught of excessive amounts of information. The participant who referred to this reflected that it was overwhelming to remember all of the competing demands of the role as an OR nurse. This participant also admitted that her role as an OR nurse was relatively new, a factor which likely added to 
concern about safety as evidenced by the following quote: "I'm concerned about being new to where I am, doing all the modules and stuff that they have on the computer, a lot of the focus is on timeout and fire safety, making sure that's part of the timeout, the focus that I see concerning is correct site, and patient positioning." The concept of infobesity also emerged as a concern in the pilot study, which was conducted one year earlier.

Inconsistency of order entry was a concern for one participant as evidenced by the following quote "Everybody is supposed to do everything on a computer, but we have people that want to fax things, which is allowed. Even though we're on an electronic system, we're still allowing the paper'. This quote reflected a frustration with inconsistency of order entry and the concern that orders would be missed when there is more than one way of finding the orders.

Generalized staff complacency referred to overall nurse complacency that was evident without specifically cited reasons. One participant reported that staff members sometimes are distracted and are not as concerned with patient safety as they should as evidenced by the following quote: "I think a lot of times, I see people get distracted and they're talking or not really paying attention." While this quote referred specifically to a safety concern that one participant had, there were many references throughout the interviews that alluded to complacency impacting normalization of deviance (See Aim 2).

\section{Aim 2) Exploration of Normalization of Deviance in the OR}

Seven out of ten participants contributed to evidence that normalization of deviance exists in the OR. Although the verbiage "normalization of deviance" was not applied to any of the questions to reduce influencing participant responses, the participants described normalization of deviance as they spoke about practices in their 
ORs. Quotes to support the evidence of normalization of deviance include "When you're a new nurse, everything is new, and everything is shiny, but if you've been in the OR for a number of years, it starts to become so mundane" and "I think sometimes people [OR nurses] get comfortable and don't watch with their tech like counting sponges or needles." Additional quotes to support evidence of normalization of deviance are presented in Table 4.

\section{Aim 3) Perceived Barriers and Facilitators to Adhering to Safety Standards}

As interviews progressed, perceived barriers and facilitators for adherence to safety standards were uncovered. Perceived barriers, which were reasons for normalization of deviance, were represented by the following five themes: productivity pressures, generalized complacency, complacency related to length of experience, social pressures, and negative acculturation. Quotes that support these themes are presented in Table 5. Facilitators to the adherence to safety standards included OR nurse engagement and supportive managerial relationships with OR nurses. Quotes that illustrate facilitators are presented in Tables 6 .

Perceived barriers to adhering to safety standards and reasons for normalization of deviance

\section{Theme 1: Productivity Pressures}

Defined as an increased emphasis on the quantity of services provided over the quality in the interest of the generation of revenue (Wilbanks et al., 2018), seven out of ten participants indicated that productivity pressure was a reason for normalization of deviance. The following quotes from 2 OR nurses illustrated how OR nurses' practice of deviating from safety standards has become normalized due to pressures to work faster: 
"At some facilities, they'll [OR nurses] cut corners to try to make that productivity" and "You're not being supported to do the right thing, and they just kind of turn their head because they want quick turnover".

\section{Theme 2: Generalized Complacency}

Three out of ten participants contributed to a theme of generalized complacency. While no specific reason for this was presented, participant quotes indicated that OR nurses had lulled themselves into a space of perceived safety because adverse events are not frequent occurrences. The practices reflective of a generalized complacency included the counting process as evidenced by the following quote:

Absolutely, yes. [some] might cut some corners. Right, they're so sure of themselves that [they think] I got 10 sponges on the back table, I don't really need to count. I can just look and see. My scrub tech, I trust her. I guess they feel like 'this is just another patient; this is just another knee scope'.

Additional practice areas that illustrated a complacent approach included tasks associated with the prevention of surgical site infection as well as the patient care required in the immediate post-operative period.

\section{Theme 3: Complacency related to Length of Experience}

In this theme, specific examples of complacency related to OR nurses' length of experience were presented by six out of ten participants. Although two participants cited experience as a strength, asserting that when nurses had more experience, it gave them more confidence and voice to speak up for safety, a majority of participants spoke to the complacent attitude associated with length of experience was reflected in this exemplary quote: 
They [OR nurses] work in the OR for 15-20 years, and they've done something this way, and then you have new management come in, and that they want to adhere by this certain policy that AORN sets or that the hospital sets, and the nurses just get upset and say, 'I'm going to continue my way' and they don't change their practice.

\section{Theme 4: Social Pressures}

Four out of ten participants described social factors that influenced their practices. For example, one participant indicated that OR nurses who take steps to meticulously follow safety standards practices can perform contrary to the prevailing norm, which may result in a social ostracization and incivility. The following quote illustrates incivility from a team member when an OR nurse adheres to the count policy "I get a dirty look if I ask for another count to be done".

OR nurses may perceive the adoption of substandard safety practices as a means to assimilate into the workplace culture. In addition, OR nurses favored assimilation because it was associated with the avoidance of potential incivility. Quotes that illustrate this phenomenon include "They're trying to make the physicians happy, and they're trying to be the staff's friend, and they're not adhering to the policy. I've seen it happen" and "They're more worried about being liked rather than being a patient advocate regarding safety because they're new, so they want people to like them."

Described incivility is sourced from various team members, such as surgeons, managers, and peers. Incivility appeared embedded within productivity pressure as evidenced in the following quote: "It felt like a struggle at my job to still count and check 
pans. That is important, but I felt that it displeased my manager, because she would tell me I had slow turnover times."

\section{Theme 5: Negative Acculturation}

Acculturation refers to a general assimilation to a dominant culture (Cramm \& Nieboer, 2019). It plays a role in OR nursing because of the uniqueness of the job, and many new OR nurses depend heavily on those who precept and guide them.

Acculturation has both positive and negative aspects; however, when the dominant adopted practice is substandard behavior, negative enculturation occurs. Two out of ten participants indicated that negative enculturation can be a barrier to safety standards. This is especially true with new OR nurses on orientation because they are relativity dependent on preceptors for guidelines on practice. Therefore, if a new nurse is not guided to follow safety stands wholly, substandard practices will become normalized for the new employee.

I do think it might be lack of education and who is precepting them, because if you get a new scrub tech / nurse with a seasoned scrub tech/nurse who doesn't necessarily follow the correct practice, then the new orientee is going to follow along in their path and gain what they learned from them.

Figure 1

Barriers to adhering to safety standards/Reasons for normalization of deviance as described by OR nurses 


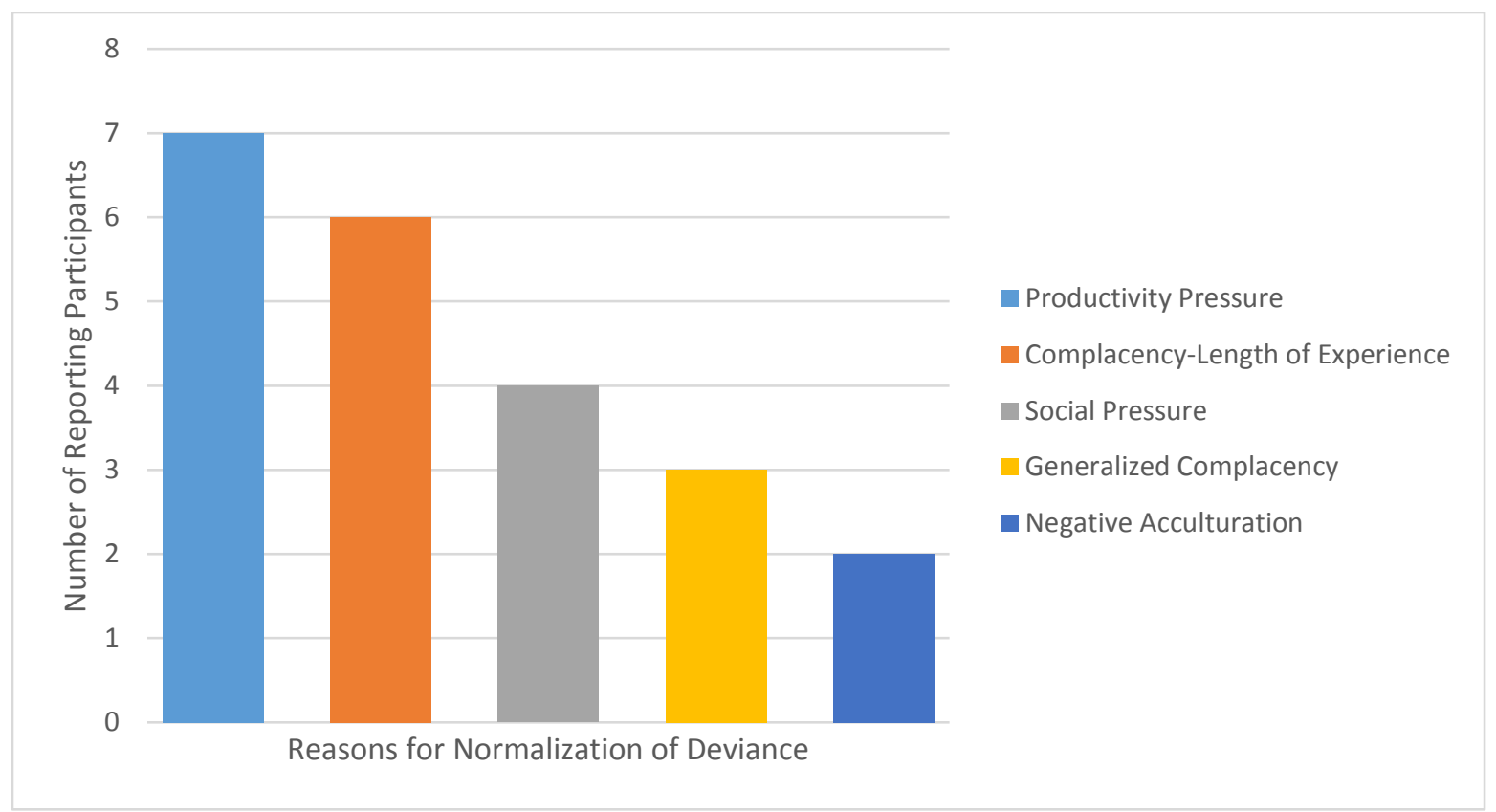

\section{Additional barriers to adhering to safety standards}

Disconnection from direct consequences was raised as an additional perceived barrier to adhering to safety standards and a reason for normalization of deviance. While it was coded as such, it was not categorized a theme because it was presented only once during the interviews. Adverse events, such as positioning injuries, are not communicated with OR nurses, as in this example, "We don't ever see any positioning injury [data]. None of that is brought to our attention, like when we have monthly meetings, none of that is shared with staff at all." The participant implied that nurses may be lulled into a sense of complacency because the sense of peril is assuaged when nurses are not informed about adverse events and consequences of behavior.

Perceived facilitators to adhering to safety standards and protective factors against normalization of deviance

\section{Theme 1: OR Nurse Engagement}


Nine out of ten participants indicated that nurse engagement was a prominent facilitator to OR nurses' adherence to safety standards. The following quote illustrates the reported positive association between OR nurse engagement and patient safety: "Absolutely, 100\%. They [engaged nurses] would [practice more safely], because if you have somebody that's more engaged that is not just there for a paycheck,... If they're engaged, you're going to have patient safety outcomes as a priority." Further quotes that contribute to the positive association between OR nurse engagement and patient safety are presented in Table 6. Participants asserted that when nurses are engaged, they are happier with their work, they pay attention to details, and they make better safety practice decisions compared to nurses who are not engaged. Furthermore, participants alluded that OR nurses did not require clinical experience to be engaged.

\section{Theme 2: Supportive Managerial Relationships with OR nurses}

Each participant was asked to describe the relationship between OR nurses and administration/ management personnel. Three out of ten participants reflected that they enjoyed supportive relationships with their managers, and it was inferred that positive relationships facilitated the adherences to safety standards and promoted OR nurse engagement:

I'm more engaged in the facility where I am now. The management is amazing. It's the best place I've ever worked. I enjoy getting up in the morning. I enjoy going and meeting my patients. It seems like if management cares more about me, I care more about my patients. I'm happy to get up and go to work and take care of the patients that I do and do the cases that I do. And everybody there feels the same way, so everybody's excited to come to work. 
While it was gleaned that OR nurses felt more supported and engaged by positive managerial support, there was evidence that negative relationships exist. For example, six out of ten participants inferred that relationships with management was fragmented and that OR nurses perceived that their managers did not understand the challenges of the OR nurse role. For example, "I don't think they [managers] know everything that goes on; they just see a start time and an end time. They don't really see what is happening; they don't understand the whole scope of care" and "I don't think they [managerial personnel] have a clue about it [the challenges]." Additional quotes illustrating fragmented relationships between OR nurses and their managers are illustrated in Table 7.

\section{Discussion}

Normalization of deviance is stealth in character because it can appear innocuous for an extended period. It is characterized by employees adjusting their behavior to fit a process that is perceived to be more efficient and logical; employees speciously conclude that deviation is acceptable because what can go wrong typically does not (Price \& Williams, 2018). The concept remains relatively new in healthcare, and the exploration of such is key to its acknowledgement, which is a crucial first step to mitigating its perilous nature.

The study's findings had some commonalties and contrasts with the findings from a pilot study, which was conducted one year earlier. The themes that emerged as reasons for normalization of deviance in the pilot study were as follows: productivity pressures, generalized complacency, experience-related variation, infobesity, and workplace incivility. While productivity pressure and generalized complacency emerged from both the pilot study and the current study, there were some variations between other themes 
from both studies. For example, experience-related variation was a reference to length of experience being an influencing factor over the adherence to safety standards. In the pilot study, most of the participants referred to length of clinical experience as a strength, specifically in relation to the confidence to speak up for safety, a trait that may accompany those who have more clinical experience. However, most of the participants in the current study indicated that length of experience was not a strength and was associated with lax attitudes towards safety. In addition, information overload, also known as "infobesity," was not a theme identified in the current study as a reason for normalization of deviance; however, it was presented by one participant as a safety concern in the current study. Workplace incivility was noted in both studies, but the theme was labeled as social pressure in the current study because it incorporated other social factors, such as a desire to fit in with the prevailing culture. Negative acculturation emerged from the current study as a reason for normalization of deviance but it was not identified in the pilot study.

Productivity pressure was by far the most prevalent theme that was uncovered during the interviews, and it presented as both a safety concern and a barrier to safety standards adherence, leading to normalization of deviance. In addition, it was a factor that was embedded in the theme of inadequate staff to support workload as a safety concern, and it was embedded within the theme of social pressures as a reason for normalization of deviance.

The study results supported the inference that OR nurses sometimes make attempts to reduce the turnover time by taking shortcuts on safety standards between surgical cases to ease pressure stemming from surgeons, managers, and even peers. The 
melding of productivity pressure and social pressure is congruent with Dekker's (2011) suggestion that deviation from safety standards can occur because of factors, such as time, cost, and peer pressure. As the theme of social pressure with an underlay of productivity pressure was presented as a barrier to maintaining safety standards, it is evident that an environment of respect and civility should be fostered to promote a healthy work environment and one that primarily focuses on patient safety. In addition, nurses should be able work in an OR environment that discourages incivility or any other kind of social pressures to prevent assimilation into a negative culture that deviates from safety standards. The OR nurse role incorporates a myriad of tasks, each of which can carry grave safety implications, and each needs to be done meticulously every time to assuage the risk for surgical patients. Therefore, when OR nurses take the time to adhere to safety standards, they should not be the subject of incivility from other team members.

Possible reasons that productivity pressure appeared to be a ubiquitous force is the OR's prevalent role as a revenue generator as well as steep operating costs. The OR has been described as the "financial hub" of any hospital" (Rothstein \& Raval, 2018, p 79), and it can generate up to $65 \%$ of hospital profit margins (Peters et al., 2018). In addition, it is estimated that OR operating costs are between $\$ 15.00$ and $\$ 50.00$ per minute (Kaye et al. 2015). These are factors that can place an inordinate pressure on OR nurses to complete tasks hurriedly, a practice that may drive them to migrate past the boundaries of what is deemed to be safe (Amalberti et al, 2006; Harvey \& Sotardi, 2017).

Within the past decade, ORs have adopted manufacturing industry standards to streamline efficiencies, many of which include reducing waste, standardizing production steps, and lowering personnel costs (Rothstein \& Raval, 2018); however, OR units are 
unlike manufacturing industries due to the extreme variability and unpredictability that is typically presented in the OR. For example, the OR is a multi-layered environment comprised of complex social interactions within a multi-disciplinary team with expectations to perform a variety of high-risk invasive procedures. In addition, surgical patients introduce a myriad of unpredictability, such as existing comorbidities that may affect the preparation for surgery or even a last-minute cancellation. Therefore, it is unrealistic to apply manufacturing industry efficiency standards to an environment that carries vast amounts of unpredictability and human risk.

The prevalence of productivity pressure that is placed on OR nurses needs to be highlighted to illustrate to management what the dangers of this kind of pressure has on patient safety. While fiscal responsibilities and efficiencies are warranted in the OR, the pressure to work faster with fewer resources may encourage inattention to precision and adventitious deviance from safety standards, which can set the stage for patient harm and stymie a culture of safety.

Generalized complacency as well as complacency related to length of experience were two separate themes that emerged from the data analysis. Participants did not indicate that OR nurses had poor intentions for patient safety; rather, this substandard safety practice was a normalized behavior that had occurred over time, specifically because the risk of adverse events appeared to be miniscule or absent. While generalized complacency, unrelated to length of clinical experience, as a reason for normalization of deviance was reflected during the interviews, complacency specifically related to length of experience was reported by twice as many participants. Length of OR nurse experience may be advantageous due to a potentially higher confidence levels coupled 
with an ability to speak-up for safety associated with experience; however, it did not appear that OR nurses' length of experience was protective against normalization of deviance in the OR. This study finding was somewhat incongruent with Benner's (1984) Novice to Expert theory, which suggests that nurses gain valuable skills as their experience increases, which increases their clinical decision-making abilities consistent with sound judgment and safety.

The theme of negative acculturation was an indication that OR nurses should be formally prepared for and supported in the role of precepting new OR nurses with a focus on patient safety. OR nurse educators can be instrumental in providing formal checkoff competency forms and guidelines that support OR nurse preceptors. Educator support can ensure that new OR nurses are being guided to adhere to safety standards, preventing new OR nurses from adopting substandard safety practices from preceptors.

In addition to the themes outlining the reasons for normalization of deviance that were gleaned from the study, one participant inferred that there is a lack of transparency in reporting and discussing adverse events in the OR, implying a lack of connection between the consequences of poor practices and negative patient outcomes. While disconnecting consequences from actions was not considered a theme in this study, the inference of the possibility of such a disconnection is a troubling sign that this issue is unique to OR nursing. For instance, surgical patients are in the OR for a relatively short period, and adverse events that are potentially related to substandard nursing practice may not be apparent immediately. For example, adverse events related to inadequate care during patient positioning under general anesthesia, surgical site infections related to substandard aseptic technique, or unintentional retainment of a foreign object related to 
poor attention during the count process, and not be acknowledged until later time, or not at all. The relative lack of awareness of adverse event consequences can be problematic because it can blunt OR nurses' perception of risk, which is a factor that can encourage normalization of deviance.

In relation to facilitators to safety standards adherence, OR nurse engagement emerged as a prevalent characteristic fortifying OR nurses against pressure to deviate from safety standards. This specific finding is congruent with past studies that examined relationships between nurse engagement and safety (Salanova et al., 2003; Sonnentag, 2003; Brooks Carthon, et al., 2019). None of these studies however focused on OR nurses or the OR setting.

Supportive managerial relationships with OR nurses also emerged as a facilitator to the adherence to safety standards and a protective factor against normalization of deviance. This finding aligns with findings of Price and Williams' (2018) indicating that leadership involvement has a pertinent role in patient safety, and unintended consequences of poor leadership affect patient safety negatively. In addition, Harvey and Sotardi (2017) suggested that risks to patient safety can be mitigated when those in leadership positions examine policies and processes with input from direct-care employees in a non-punitive and supportive environment. They also suggested that feedback from direct-care employees was a powerful conduit to understand the basis for why standards are not followed wholly. Therefore, relationships between OR nurses and management need to be fortified and energized with respect and understanding with a goal of nurturing a healthy work environment that promotes OR nurse engagement. 
Transparency is another benefit of improved relationships between OR nurses and their managers. For example, most participants indicated their managers did not understand the challenges that OR nurses face. Participants suggested that managers placed unrealistic expectations on OR nurses to perform faster without the necessary support. It is therefore essential for OR managers to acknowledge challenges that OR nurses encounter, and it is imperative that barriers to safety standard adherence and reasons for normalization of deviance be acknowledged and understood, so that solutions can be offered. For example, the expectation to adhere to scrupulous and necessary safety standards should be paired with enough support to meet productivity goals. In addition, there should be open discussions relating to adverse events, deviations from safety standards, and near- misses in a non-punitive, civil environment so that OR nurses can feel empowered to defend safety standards and be more aware of the potential and actual adverse events.

\section{Limitations}

Limitations to the study include participants working within the same metropolitan, geographical location, which may affect the transferability of the findings to other geographical areas. In addition, some reasons for normalization of deviance were identified and coded, but they were not categorized as themes because of the infrequency of quotes to support the reasons, which is an indication that data saturation may not have been reached in its entirety.

\section{Conclusions}

The OR is a high-risk environment, and although adverse events do not occur frequently, OR nurses have safety concerns. Some of the most prevalent safety concerns 
reported were productivity pressures and inadequate staff to support workload, while other concerns included "infobesity," inconsistency of order entry, and general complacency of staff. There is evidence that normalization of deviance exists in the OR, and five primary reasons for the deviation identified in this study were: productivity pressures, generalized complacency, complacency related to length of experience, social pressures, and negative acculturation. Facilitators to the adherence to safety standards included OR nurse engagement and a positive supportive relationship with managerial personnel.

Evidence of normalization of deviance within the OR should serve as a warning light on the dashboard of OR safety, and one that signals a problematic system deserving of attention before surgical patients' lives and wellbeing are jeopardized. This phenomenon must become a priority when discussing how to maintain safety standards for vulnerable surgical patients who rely on their OR teams to maintain their safety and wellbeing during the surgical period. Finally, it is imperative to encourage an environment that supports adherence to OR safety standards, such as the employment of strategies to promote and sustain OR nurse engagement and efforts to foster supportive, transparent, managerial relationships with OR nurses. 
Table 2. Interview Questions

\begin{tabular}{|c|c|}
\hline & Interview Questions \\
\hline 1 & In your practice, what do you see as the top safety concerns at your OR department? \\
\hline 2. & Describe areas of OR personnel practice that you consider unsafe or potentially set the stage for an adverse event to occur? \\
\hline Probe & $\begin{array}{l}\text { There are specific guidelines in place to help prevent two of the surgical "Never Events" include: Wrong Site/Person } \\
\text { Surgery and Retained Foreign Objects. Do you have examples where you have seen how personnel may increase patients' } \\
\text { vulnerability in relation to these two never events? }\end{array}$ \\
\hline 3. & Describe any other concerns that you have relating to patient safety in the OR? \\
\hline Probe & Describe how you think personnel practices from a safety standpoint? \\
\hline Probe & How do you think the length of experience affect how personnel practice from a patient safety standpoint? \\
\hline Probe & $\begin{array}{l}\text { How do you think overall nurse engagement, for example, nurses who are absorbed in their work or those who have a } \\
\text { positive attitude about their work, affect how nursing personnel practice from a patient safety standpoint? }\end{array}$ \\
\hline 4. & Tell me what you think are facilitators to adhering to OR standards and protocols? \\
\hline 5. & Describe any barriers that you perceive as obstacles that impede safety practices? \\
\hline Probe & Describe how you think productivity pressures may affect practice? \\
\hline 6. & Describe the relationship between OR nurses and managers where you work \\
\hline Probe & How do you think administration/managers affect safety practices? \\
\hline
\end{tabular}


Table 3. Quotes supporting primary safety concerns in the OR: Practicing OR nurse perspectives (Study Aim 1)

\section{Primary Safety Concerns in the OR from the Perspective of Practicing OR RNs}

\begin{tabular}{|c|c|}
\hline $\begin{array}{l}\text { Theme 1: } \\
\text { Productivity } \\
\text { Pressures }\end{array}$ & $\begin{array}{l}\text { "Everything is all about getting patients in (the OR) exactly on time, turnover time, but the cases get more } \\
\text { complex, but we don't get any more time to prepare, so I feel like where that corner gets cut is doing } \\
\text { assessments, doing all the pre-work we need before patients come into the OR." }\end{array}$ \\
\hline \multirow{9}{*}{$\begin{array}{l}\text { (Reported by } \\
\text { eight of out ten } \\
\text { participants) }\end{array}$} & "Personally where I work, it’s all about money and less about patient care now." \\
\hline & "The top patient concern (that) is most prevalent is time efficiency versus just general good medicine." \\
\hline & $\begin{array}{l}\text { "We're (OR nurses) constantly being made to cut corners for efficiency versus reviewing patient records, } \\
\text { making sure that we have everything, that the patient is stable, all of our ducks lined up in a row." }\end{array}$ \\
\hline & $\begin{array}{l}\text { "I see more and more responsibilities being loaded on to OR nursing, which further decreases that time that we } \\
\text { have to prepare for our cases, check our patients in, make sure there's a continuity of care between pre-op and } \\
\text { intraop." }\end{array}$ \\
\hline & $\begin{array}{l}\text { "They're (managers) pushing you to get more cases done; surgeons are pushing you, and administration is } \\
\text { pushing you" }\end{array}$ \\
\hline & $\begin{array}{l}\text { "We've never had a fire, but not allowing the 3-minute drying time rule on skin preps, because nurses are feeling } \\
\text { rushed by the surgeons for time is concerning;", }\end{array}$ \\
\hline & "Efficiency has kind of given way to patient safety." \\
\hline & "I'm worried about med errors a lot of the time ....just because I'm feeling rushed." \\
\hline & "I'm concerned about patient positioning...because it's such a fast area" \\
\hline
\end{tabular}




\begin{tabular}{|c|c|}
\hline & "I've seen it (productivity pressure) affect people \\
\hline $\begin{array}{l}\text { Theme 2: } \\
\text { Inadequate staff } \\
\text { to support } \\
\text { workload } \\
\end{array}$ & $\begin{array}{l}\text { "The top patient safety (concern) is patient positioning on a narrow bed and somebody falling off. At our facility, } \\
\text { we don't have a whole lot of staff, so trying to bring the patient into the bed by yourself always concerns me." }\end{array}$ \\
\hline \multirow[t]{3}{*}{$\begin{array}{l}\text { (Reported by } \\
\text { four out of ten } \\
\text { participants) }\end{array}$} & $\begin{array}{l}\text { "The lack of staffing that we have to help support the amount of patients we have because a lot of times we're } \\
\text { lifting patients without having enough staff members. So, it's potential risk for a patient to fall down or fall off } \\
\text { the OR table and transition." }\end{array}$ \\
\hline & "We don't have as many people as we should right now" \\
\hline & $\begin{array}{l}\text { "Room turnovers (time), are tough because our staffing situation right now is not ideal as far as people that we } \\
\text { hire to turn over rooms and stuff" }\end{array}$ \\
\hline \multicolumn{2}{|l|}{$\begin{array}{l}\text { Additional Safety } \\
\text { Concerns }\end{array}$} \\
\hline a) Infobesity & $\begin{array}{l}\text { "I'm concerned about being new to where I am, doing all the modules and stuff that they have on the } \\
\text { computer, a lot of the focus is on timeout and fire safety, making sure that's part of the timeout, the focus that I } \\
\text { see concerning correct site, and patient positioning." }\end{array}$ \\
\hline $\begin{array}{l}\text { b) Inconsistency } \\
\text { order entry }\end{array}$ & $\begin{array}{l}\text { "Everybody is supposed to do everything on a computer, but we have people that want to fax things, which is } \\
\text { allowed. Even though we're on an electronic system, we're still allowing the paper." }\end{array}$ \\
\hline $\begin{array}{l}\text { c) } \begin{array}{l}\text { Generalized } \\
\text { complacency } \\
\text { of staff }\end{array}\end{array}$ & "I think a lot of times, I see people get distracted and they're talking or not really paying attention" \\
\hline
\end{tabular}


Table 4. Quotes supporting the occurrence of normalization of deviance in the OR (Study Aim 2)

\begin{tabular}{|c|c|}
\hline \multicolumn{2}{|c|}{ Normalization of Deviance in the OR } \\
\hline $\begin{array}{l}\text { Quotes } \\
\text { supporting } \\
\text { the existence } \\
\text { of } \\
\text { normalization } \\
\text { of deviance in } \\
\text { the OR }\end{array}$ & $\begin{array}{l}\text { "When you're a new nurse, everything is new, and everything is shiny, but if you've been in the OR for a number of } \\
\text { years, it starts to become so mundane, and people just start cutting corners a bit here and there." }\end{array}$ \\
\hline \multirow{7}{*}{$\begin{array}{l}\text { (Reported by } \\
\text { seven out of } \\
\text { ten } \\
\text { participants) }\end{array}$} & "We all know it's not right, but it just happens" \\
\hline & $\begin{array}{l}\text { "I think sometimes people [OR nurses] get comfortable and don't watch with their tech like counting sponges or } \\
\text { needles." }\end{array}$ \\
\hline & $\begin{array}{l}\text { "Sometimes it's like you should just have a blind faith that she's doing her job and keeping track of everything. I've } \\
\text { had to dig through garbage looking through for needles and sponges, so I just don't think people think about that; I } \\
\text { think sometimes it becomes a casual environment." }\end{array}$ \\
\hline & $\begin{array}{l}\text { "I actually felt like a black sheep because I would count instruments and check wraps before getting the patient. It's } \\
\text { so fast-paced, you don't really have the time to count with your tech" }\end{array}$ \\
\hline & $\begin{array}{l}\text { "The more you're being pushed, the more you just do it because there is no one else to do it. I think a lot of times, } \\
\text { they're just 'oh well, this is how things are done"" }\end{array}$ \\
\hline & $\begin{array}{l}\text { We've never had a fire but our preps are not used the correct way; not allowing the 3-minute rule, because nurses } \\
\text { are rushed for time" }\end{array}$ \\
\hline & $\begin{array}{l}\text { "If we work together, I trust you; I know that you've never had anything bad happen; so I know you haven't } \\
\text { counted needles, but I make the assumption [that nothing bad will happen]. It's just normal routine; it's the same } \\
\text { thing for every case". }\end{array}$ \\
\hline
\end{tabular}


Table 5

Quotes supporting perceived barriers to adhering to safety standards in the OR; Reasons for normalization of deviance (Study Aim 3)

\section{Perceived Barriers to Adhering to Safety Standards in the OR (Reasons for Normalization of Deviance)}

\begin{tabular}{l|l} 
Theme 1 & "I would say 95\% of our nurses fall into the pressure because if you don't, then they [managerial personnel] will call
\end{tabular}

Productivity into your room and ask 'Why aren't you ready? What are you doing? We need to hurry up and get these cases

Pressures $\quad$ done'... and that makes their [OR nurses] anxiety peak; things haven't been counted, and you're trying to hurry up and count and put drugs on the field. There's just a lot of room for error because you can't do five things at once

while the patient's in the room and be safe at the same time"

(Reported by "In the environment that I was in where you're not being supported to do the right thing, and they just kind of turn seven out of their head because they want quick turnover, so it's like they're not enforcing pans getting checked or safety issue

ten with power cords"

participants)

"There's pressure [from] the physicians who push you to get the patient back [to the OR] when you are not ready. I

do feel like sometimes [nurses] cave and run out to get the patient in the room- there's noise and confusion, and a lot going on"

"It's like we're trying to get this case going, so high priority is not checking that pan to see if it's sterile."

"Some nurses really don't do the 3 minutes [referring to waiting for alcohol-based skin preps to dry] like they're supposed to because of time and the surgeon is in the room looking, waiting, and wanting to start"

"The timeout thing, they [OR nurses] are just trying to make surgeons happy [by hastening the process] and not making patient safety their top priority"

"We've never had a fire but our preps are not used the correct way; not allowing the 3-minute rule, because nurses are rushed for time"

"They're pushing to get more cases done, and the surgeons are pushing you, the administration is pushing you"

"When you're trying to turn a room over quickly, I'm saying [the cleaning of] the underneath stuff [may not get done properly]

"At some facilities, they'll [OR nurses] cut corners to try to make that productivity, and I've seen that." 


\begin{tabular}{|c|c|}
\hline $\begin{array}{l}\text { Theme 2: } \\
\text { Generalized } \\
\text { Complacency }\end{array}$ & $\begin{array}{l}\text { "Oh, I think absolutely, yes [that some] might cut some corners. Right, they're so sure of themselves that [they think] } \\
\text { I know they got } 10 \text { sponges on the back table, I don't really need to count. I can just look and see. My scrub tech, I } \\
\text { trust her." I guess they feel like 'this is just another patient. This is just another knee scope."” }\end{array}$ \\
\hline \multirow{5}{*}{$\begin{array}{l}\text { (Reported by } \\
\text { three out of } \\
\text { ten } \\
\text { participants) }\end{array}$} & "People just get used to the way they've always done things" \\
\hline & $\begin{array}{l}\text { "I think sometimes it can be a safety thing because people are coming in the room }[O R] \text { and they're being loud and } \\
\text { talking, and you still have a patient in there; it could cause a distraction with people waking up from surgery." }\end{array}$ \\
\hline & $\begin{array}{l}\text { At the other place I worked a lot of people, it's things getting overlooked, like a dirty light handle left on, things not } \\
\text { getting wiped down. We did a lot of pediatric dental, people trying to do things quickly and having blood left on } \\
\text { stuff in a previous case." }\end{array}$ \\
\hline & "I think sometimes people get comfortable and don’t watch with their tech like counting sponges or needles" \\
\hline & "Sometimes people get comfortable and don't watch with the tech counting sponges and needles" \\
\hline $\begin{array}{l}\text { Theme 3: } \\
\text { Complacency } \\
\text { related to } \\
\text { length of } \\
\text { experience }\end{array}$ & $\begin{array}{l}\text { "They [OR nurses] work in the OR for } 15-20 \text { years, and they've done something this way, and then you have new } \\
\text { management come in, and that they want to adhere by this certain policy that AORN sets or that the hospital sets, } \\
\text { and the nurses just get upset and say, 'I'm going to continue my way' and they don't change their practice" }\end{array}$ \\
\hline \multirow[t]{2}{*}{$\begin{array}{l}\text { (Reported by } \\
\text { six out of ten } \\
\text { participants) }\end{array}$} & $\begin{array}{l}\text { "I see some nurses that have been there a really long time, not necessarily just ones that I have experienced, but some } \\
\text { of them I think do get a little lax with counts." }\end{array}$ \\
\hline & $\begin{array}{l}\text { "It just happens because nurses who've been working for a long time sometimes get lax or complacent just because } \\
\text { honestly, they might be a little burnt out. And they feel like they're tired, and it's easier to do it their way instead of } \\
\text { doing it the way that you're supposed to according to policy." }\end{array}$ \\
\hline
\end{tabular}




\begin{tabular}{|c|c|}
\hline & $\begin{array}{l}\text { "A lot of times that's more of the seasoned nurses that are used to doing things the way they've always done them } \\
\text { and not wanting to change. I think it's a little dangerous." }\end{array}$ \\
\hline & $\begin{array}{l}\text { "Well, to go back where I worked before, I know one of the techs had been a tech for a long time, but I would see } \\
\text { her in the hallway carrying an instrument pan on its side. She would open up pans without checking the wrappers on } \\
\text { them." }\end{array}$ \\
\hline & $\begin{array}{l}\text { "I think it's hard for anyone who has been doing something that long to adapt to a new practice. But I do think it affects } \\
\text { patient safety when you don't adhere to a policy" }\end{array}$ \\
\hline & "You have the people who are ready for retirement, and I think some just tune out and it becomes 'I'm sick of this'." \\
\hline & "You have nurses who are seasoned and just don't really want to change what they are doing" \\
\hline $\begin{array}{l}\text { Theme 4: } \\
\text { Social } \\
\text { Pressures }\end{array}$ & $\begin{array}{l}\text { "They're more worried about being liked rather than being a patient advocate regarding safety because they're new, } \\
\text { so they want people to like them." }\end{array}$ \\
\hline \multirow{5}{*}{$\begin{array}{l}\text { (Reported by } \\
\text { four out of } \\
\text { ten } \\
\text { participants) }\end{array}$} & $\begin{array}{l}\text { "They're trying to make the physicians happy, and they're trying to be the staff's friend, and they're not adhering to } \\
\text { the policy. I've seen it happen. " }\end{array}$ \\
\hline & "I get a dirty look if I ask for another count to be done" \\
\hline & $\begin{array}{l}\text { "I actually felt like the black sheep, because I would count my instruments and check the wrappers before I would go } \\
\text { get the patient, and they would be pushing me to go get the patient. So, yeah, I did feel a little like what I was doing } \\
\text { was out of the norm." }\end{array}$ \\
\hline & $\begin{array}{l}\text { "Yeah, there was a disconnect, and a lot of favoritism...like the social part of it seemed to be more important than } \\
\text { prioritizing things that were going on with patients and the cases." }\end{array}$ \\
\hline & $\begin{array}{l}\text { "I've just been in a situation where one particular surgeon was rushing me, and I made a mistake because of it. I } \\
\text { mean, the patient ended up being fine, but it was scary. I need a second to figure out what I was doing. And he was } \\
\text { yelling, and it was very intimidating, and that's not fair to the patient for him to be behaving that way because that } \\
\text { affected the patient" }\end{array}$ \\
\hline
\end{tabular}




\begin{tabular}{|l|l|}
\hline & $\begin{array}{l}\text { "It felt like a struggle at my other job to still counting and checking pans. That is important, but I felt that it } \\
\text { displeased my manager, because she would tell me I had slow turnover times." }\end{array}$ \\
\hline $\begin{array}{l}\text { "We have some doctors who want what they want; 'they want it yesterday' kind of mentality. And if they don't get it } \\
\text { immediately, it's gets stressful really quick. All of a sudden when someone is yelling at you, you're like 'OK"" }\end{array}$ \\
$\begin{array}{l}\text { Theme 5: } \\
\text { Acculturation }\end{array}$ & $\begin{array}{l}\text { "I do think it might be lack of education and who is precepting them, because if you get a new scrub tech/nurse with } \\
\text { a seasoned scrub tech/nurse who doesn't necessarily follow the correct practice, then the new orientee is going to } \\
\text { follow along in their path and gain what they learned from them. " }\end{array}$ \\
\hline $\begin{array}{l}\text { (Reported by } \\
\text { two out of ten } \\
\text { participants) }\end{array}$ & $\begin{array}{l}\text { "And then we had some nurses that were new to the OR, and they're following what other people are doing, like not } \\
\text { counting until they're in the room, or just seems really lax, or not knowing that you need to check the wrappers" }\end{array}$ \\
\hline
\end{tabular}

Table 6

Quotes supporting perceived facilitators to the Adherence of Safety Standards/Protective factors against normalization of deviance (Study Aim 3)

\begin{tabular}{|l|l|l|}
\hline Nurse Engagement and its Role in Safety Practices \\
\hline $\begin{array}{l}\text { Theme 1 } \\
\text { OR Nurse } \\
\text { Engagement }\end{array}$ & $\begin{array}{l}\text { "Oh, engagement is important 100\% because if you aren't wanting to be at work, you might miss a step. If you're } \\
\text { tucking arms and you just want to hurry up and get them tucked and you don't use a grate to protect their elbows or } \\
\text { something, just little things that could definitely affect patient safety. And counting, just wanting to hurry up and get } \\
\text { it done so you can get these cases started and not worrying about opening up the packs, and things like that. So, I } \\
\text { definitely can see that engagement influences safety." }\end{array}$ \\
$\begin{array}{l}\text { (Reported by } \\
\text { nine out of } \\
\text { ten } \\
\text { participants) }\end{array}$ & "I feel like if you're engaged, certainly, I feel like a person would be more willing to do things the right way" \\
\hline
\end{tabular}




\begin{tabular}{|l|l|}
\hline & $\begin{array}{l}\text { "Yeah, if your mind is elsewhere, if you're not happy in what you're doing and it's a sense of doom, then it's not } \\
\text { going to be good outcomes that happen. I think if you're focused and your employees are happy, then that shows on } \\
\text { into the patients. } \\
\\
\text { "Yeah, I think if you're not engaged, you probably honestly don't care as much, and when you don't care as much, it } \\
\text { affects your patients, I think." } \\
\text { "Absolutely, 100\%. They (engaged nurses) would (practice safer), because if you have somebody that's more engaged } \\
\text { that is not just there for a paycheck, or they're not just there because nurses make a decent salary. If they're there for } \\
\text { the right reasons and they're engaged, you're going to have patient safety outcomes as a priority, for sure 100\%" }\end{array}$ \\
\hline & "You've got to enjoy what you're doing and be concerned about the procedure and outcome for that patient. \\
\hline & $\begin{array}{l}\text { "Engagement helps you be more conscientious about what you're doing". Certainly, if you're engaged in something, } \\
\text { you care about what you're doing... and you're better at your job" }\end{array}$ \\
\hline "I think engagement definitely helps you be more conscientious about what you're doing." \\
\hline $\begin{array}{l}\text { Theme 2: } \\
\text { Supportive } \\
\text { Managerial } \\
\text { Relationships } \\
\text { with OR } \\
\text { Nurses }\end{array}$ & $\begin{array}{l}\text { "So if you have somebody coming in that's unhappy and doesn't want to be there, then that goes onto the patient; it } \\
\text { doesn't stop. It tumbleweeds from there and bad outcomes can happen, just from attitude" } \\
\text { about me, I care more about my patients. I'm happy to get up and go to work and take care of the patients that I do and } \\
\text { do the cases that I do. And everybody there feels the same way, so everybody's excited to come to work." }\end{array}$ \\
\hline $\begin{array}{l}\text { Reported by } \\
\text { three out of } \\
\text { ten } \\
\text { participants }\end{array}$ & $\begin{array}{l}\text { "I feel like we have a pretty good relationship with our management. They're very open to-like I feel like I can go } \\
\text { talk to my manager if I feel like I've made a mistake or something and I need to fess or something, I totally feel } \\
\text { comfortable doing that with my manager. She is very experienced. She was in the OR forever as a circulator, so I feel } \\
\text { like she understands how it is. And they're very open to feedback and all of that from the staff" }\end{array}$ \\
\hline
\end{tabular}




\begin{tabular}{|l|l|}
\hline & $\begin{array}{l}\text { "Yeah, it's good [relationship with management] It's kind of like sometimes I have to stop myself because of the } \\
\text { pressure that I felt from my other job, and they're not pushing me [in this current job] like that. }\end{array}$ \\
\hline & $\begin{array}{l}\text { "Our manager comes and helps us clean rooms and turn rooms over. She helps us lift patients. She helps us, she } \\
\text { maybe goes beyond what others do. So she knows what goes on." }\end{array}$ \\
\hline
\end{tabular}


Table 7

Quotes representing unsupportive managerial relationships with OR nurses

\begin{tabular}{|c|c|}
\hline \multicolumn{2}{|c|}{ Managerial Support } \\
\hline $\begin{array}{l}\text { Quotes } \\
\text { reflecting } \\
\text { negative, } \\
\text { unsupportive } \\
\text { managerial } \\
\text { relationships } \\
\text { with OR } \\
\text { nurses }\end{array}$ & $\begin{array}{l}\text { "I don't think they (managerial personnel) have a clue about it ( the challenges)" "If there is a } 30 \text {-minute surgery } \\
\text { turnover time, they may not understand that there was a gross contamination spill and if we hurried through clean- } \\
\text { up, we would jeopardize (the safety of) the next patient." }\end{array}$ \\
\hline \multirow{6}{*}{$\begin{array}{l}\text { (Reported by } \\
\text { six out of ten } \\
\text { participants) }\end{array}$} & "I think they (managers) need to come work in the OR a couple of times a year to see what it is like" \\
\hline & "I think they (managers) are not there to see what goes on in the OR" \\
\hline & "The expectations (from managers) that are put on you are unrealistic" \\
\hline & $\begin{array}{l}\text { "I think we feel like we sometimes fall on deaf ears when we talk to the person above who is over all of surgical } \\
\text { services. Administration has no clue what it's like to in surgery, I mean they don't ever really come back to surgery } \\
\text { to see what happens there" }\end{array}$ \\
\hline & $\begin{array}{l}\text { "I don't think they (managers) have a clue; they don't go in the room; they are not turning over; they don't } \\
\text { understand how it is" }\end{array}$ \\
\hline & $\begin{array}{l}\text { "I don't think they (managers) know everything that goes on; they just see a start time and an end time. They don't } \\
\text { really see what is happening; they don't understand the whole scope of care" " }\end{array}$ \\
\hline
\end{tabular}




\section{CHAPTER IV}

\section{EXAMINING FACTORS THAT PREDICT CULTURE OF SAFTEY IN THE OPERATING ROOM (OR)}

\section{Background}

A culture of safety within a healthcare facility is determined by the internalized values and creed of facility personnel, which are reflective of their behaviors, and it is associated with positive patient outcomes. Achieving and sustaining a culture of safety has particular salience in the operating room (OR) because it is a one of the most intricate and complicated work environments in healthcare. OR personnel work within a fallible system weakened by productivity pressures and team instability, factors that add risk to patient safety. Therefore, a culture of safety in the OR is critical to thwart errors in this high-risk environment, and it is imperative to examine the factors that support a culture of safety.

\section{Purpose}

The purposes of this study were to: (1) examine relationships between length of OR nurse experience, OR nurse engagement, and culture of safety in the OR, (2) examine if length of OR nurses' clinical experience and OR nurse engagement predict culture of safety in the OR, and (3) determine differences in culture of safety in the OR based on certification in OR nursing (CNOR) 


\section{Method}

A convenience sample of 96 OR nurses were recruited from an electronic and social media platform. Safety culture attitudes were measured using the Safety Attitudes Questionnaire (SAQ). Nurse engagement was measured using the Utrecht Work Engagement Scale (UWES). Length of nurse experience measured in years was collected using a demographic data collection form. Bivariate associations between the following variables were examined: length of OR nurses' clinical experience and OR culture of safety; OR nurse engagement with OR culture of safety; and length of OR nurses' experience and OR nurse engagement. A standard multiple linear regression was conducted to examine if length of OR nurse experience and OR nurse engagement were predictors of OR culture of safety. In addition, an independent sample t-test was conducted to examine if safety of culture scores were statically significantly different in those who had a CNOR compared to those without certification.

\section{Findings}

The length of OR nurse experience was positively correlated with OR culture of safety; however, the relationship was weak, and it was not statistically significant. In contrast, OR nurse engagement had a strong, statistically significant relationship with OR culture of safety, and was a significant predictor of culture of safety in the OR. Finally, OR nurses who had a CNOR certification showed a statistically significantly higher culture of safety scores compared with those without CNOR certification.

\section{Introduction}

Patient safety is the foundation for providing healthcare and has been compared to the provision of biological, physiological, and safety needs from Maslow's hierarchy 
model (Maslow, 1954; Ulrich \& Kear, 2014). Singer et al. (2009) concluded that a culture of safety within a healthcare facility was associated with higher levels of healthcare personnel safety-related behaviors and better patient outcomes, which established a link between a healthcare organization's culture of safety and patient safety.

Safety in healthcare became a prominent focus within the industry two decades ago when the Institute of Medicine's (IOM) 1999 report, To Err Is Human: Building a Safer Health System, reported that adverse events occur in up to $3.7 \%$ of annual admissions, and $13.6 \%$ of these events lead to patient mortality. The 1999 IOM report stated that errors within the healthcare industry were responsible for approximately 44,000 to 98,000 patient deaths in the United States annually (Kohn, Corrigan, \& Donaldson, 2000). The report asserted that errors were not the result of incompetent people making mistakes; rather, healthcare errors are multi-faceted and embedded within a culture.

While a culture of safety carries importance throughout the healthcare industry, it holds specific prominence in the operating room (OR) because of its informationintensive, high-risk nature. In addition, OR personnel work within a fallible system weakened by time pressures, goal conflicts, team instability, and significant authority disparity between various disciplines (Carthey, 2014). Although OR safety has been highlighted in the literature, and consequently efforts have been made to mitigate deficiencies in OR safety systems, safety violations continue to be a problem, and adverse events in the OR remain a serious threat to surgical patients (Zahiri et al., 2011; Goldenberg \& Elterman, 2019).

Surgical patients are some of the most vulnerable patients in healthcare. Due to procedural medications, anesthesia, invasive procedures, and general procedural 
restrictions applied to surgical patients, they often relinquish their independence as well as their ability to speak or act on their own behalf, thereby placing an explicit trust in the surgical team that cares for them during the operative period. The surgical team has a prodigious responsibility to be mindful stewards of safety practices for the patients in their care and achieve and sustain a culture of safety in the OR environment. The OR is a complex, high-risk environment, and safety for patients is dependent on a sound safety culture with practices that adhere to safety standards. It is imperative therefore to examine OR safety culture and associated factors.

The culture of safety in the OR should be examined before adverse events occur so that a more transparent understanding can be gleaned from processes and environmental influences that affect safety can be identified. This is in contrast to a statement made by Christian et al. (2006) when they reported that many studies of OR safety are done after an adverse event occurs. Espin et al. (2006) concluded that deficiencies in the culture of safety of ORs were problematic, and they acknowledged that while improving patient safety in the OR was a challenge, a crucial first step was to explore related and influential factors.

The purpose of this study was to examine relationships between length of OR nurse experience, OR nurse engagement, and OR culture of safety, in addition to investigate if the length of OR nurse experience and nurse engagement are predictors of OR culture of safety.

The specific aims and hypotheses pertaining to the study were to:

1) Explore the relationships between length of OR nurses' experience, OR nurse engagement, and OR culture of safety 
$\mathrm{H} 1$ : there is a positive relationship between the length of the OR nurse experience and OR culture of safety

$\mathrm{H} 2$ : there is a positive relationship between OR nurse engagement and OR culture of safety

$\mathrm{H} 3$ : there is a positive relationship between the length of the OR nurse experience and OR nurse engagement

2) Determine if length of OR nurse experience and nurse engagement predict OR culture of safety

H4: Length of the OR nurse experience and OR nurse engagement predicts OR culture of safety

3) Identify the extent that the variance in OR culture of safety is explained by the length OR nurse experience and OR nurse engagement.

4) Determine differences in OR culture of safety based on certification in OR nursing (CNOR).

\section{Background and Significance}

A culture of safety has been defined as "a set of values that are shared within an organization that relate to what is important and beliefs about how the organizational structure should be to produce behavioral norms that promote safety" (Singer, Lin, Falwell, Gaba, \& Baker, 2009, p 400). Singer et al. (2009) concluded that a culture of safety within a healthcare facility is determined by the internalized values and creed of facility personnel, which are reflective of their behaviors.

Attaining and sustaining a safety culture for healthcare gained a robust traction after the IOM's 1999 report To Err Is Human: Building a Safer Health System, which 
outlined sobering statistics on healthcare errors and adverse patient outcomes. The report was an impetus that prompted healthcare systems to examine their personnel's safetyrelated behavior (King, 2010; Kohn et al., 2000). As a result, many healthcare facilities made strides to improve safety behaviors, such as efforts to improve teamwork and to introduce transparent reporting of errors in a non-punitive manner (Espin, Lingard, Baker, \& Regehr, 2006), initiatives that were deemed crucial to modify the culture of safety and to address the sociological contributors to safety practices (King, 2010; Zahiri et al., 2011). Despite significant efforts by many healthcare organizations to improve safety behaviors, medical errors persisted, generating substantial personal and monetary cost (IOM, 2006). Brooks Carthron et al. (2019) asserted that many of the endeavors aimed at increasing safety over the past 20 years focused on checklists and improved healthcare records rather than assessing work environments and overall culture, and they asserted that more work was required to attain optimal safety standards.

The culture of safety has a distinct salience in the OR in light of the high-risk nature of the complex and multi-faceted environment (Wright, 2016; Link, 2019). ORs typically have sophisticated technology and team members comprised of different professions who work irregular hours within a stressful environment, factors that can enhance the risk for error (Ongun \& Intepeler, 2017). While efforts to mitigate risk occur in the OR, iatrogenic adverse events remain a significant threat to surgical patients (Goldenberg \& Elterman, 2019).

U.S. ORs had close to 10,000 never-events, which are occurrences that include wrong-site, wrong-procedure, wrong-person surgeries, and retained surgical items, between 1990 and 2010. Almost 7\% of these events resulted in a patient's death. In 
addition, over $\$ 1.3$ billion was paid toward malpractice claims related to those events, the payments for which did not encompass the financial strain of legal fees, patient disability, lost work time, and defamation of the surgical team and facility involved (Mehtsun, Ibrahim, Diener-West, Pronovost, \& Makary, 2013).

In 2004, the Joint Commission required the use of the Universal Protocol, a process that mandated a carefully plotted process of patient and procedure identifications prior to surgery to increase patient safety standards by avoiding the occurrence of wrong site or wrong patient procedures (The Joint Commission, 2004). However, this adopted protocol did not meet its expected intentions; the standardized protocol failed to prevent such events (Stahel, Mehler, Clarke, \& Varnell, 2009). According to Stahel et al. (2009), reasons for the failure of the Universal Protocol included inconsistencies in site-marking procedures, discrepancies in education for staff, and general deviations from the intended protocol (Stahel et al., 2009; Banschbach, 2009). King (2010) reported that although the IOM purported that system weaknesses caused errors in the OR, managers have suggested that lack of adherence to safety protocols and an etiolated safety culture were primary reasons.

While it is reasonable to suggest that the data on reported adverse events in the OR should influence the needle of the safety culture compass and subsequently guide efforts to increase or sustain a safety culture, a relative absence of reported adverse events does not necessarily determine a sound safety culture. One of the reasons for this is that currently, the reporting of adverse events in the U.S., surgical never-events or otherwise, is ambiguous and poorly understood (Austin \& Pronovost, 2015; Goldenberg \& Elterman, 2019). Challenges with reporting data are due to lack of transparency and 
suboptimal standardized national reporting systems and definitions. Specifically, prospectively collected data on the incidence of surgical never-events are limited, and many studies involve voluntary reporting to external agencies with an inherent bias (Austin \& Pronovost, 2015; Theils et al. 2015; Goldenberg \& Elterman, 2019). In addition, reporting of events can be associated with fear of litigation and potential insult to professional reputations (Goldenberg \& Elterton, 2019). Apart from adverse events, near-misses, defined as events that did not cause patient harm but had potential to do so (Goldenberg \& Elterton, 2019), are enormously challenging to capture and report adequately because of their subjective nature in addition to perception biases and difficulty of detail recollection. The lack of comprehensive data reporting of adverse events or near misses is therefore problematic because the reality that flawed safety systems may not be overt. This is subject to pacifying OR personnel into a perception that the existing safety system is more robust than is true, which could dull efforts to mitigate deficiencies in safety-related processes.

Regardless, however, of reported data, Goldenberg and Elterman (2019) suggested that efforts to improve OR safety should be channeled in proactive and prospective manners rather than reacting to the occurrence of adverse events. The culture of safety should be examined before adverse events occur so that a more transparent understanding can be gleaned from the processes, and environmental influences that affect safety can be identified.

Researchers have studied various factors that correlate with OR culture of safety, such as the complexity of facilities, the use of checklists, and safety performance behaviors (Carney, West, Neily, Mills, \& Bagian, 2010; Lepanluoma, Takala, 
Kotkansalo, Rahi, \& Ikonen, 2014; Singer et al., 2009). While valuable information has been gleaned from these studies, there has been a dearth of focus on characteristics of OR nurses in relation to a culture of safety, such as the length of clinical experience and nurse engagement. Nurse engagement is defined as a state of mind that is fulfilled and satisfied, and it is characterized by commitment and immersion in one's work (Schaufeli et al., 2002). These variables and their association with safety are illustrated in Benner's (1984) Novice to Expert model that explains how clinical experience leads to knowledge, engagement, and sound decision-making consistent with safety. Nurses who remain in their roles for longer periods have been touted to be more beneficial to their clinical environments than their less experienced peers because the knowledge and experience that is acquired over time is a key component in quality care and overall safety for patients (Benner, 1984; Riley, Dearmon, Mestas, \& Buckner, 2016).

Singer et al. (2009) suggested that there was a discrepancy between perceived culture of safety between healthcare personnel with varying lengths of clinical experience. Their study showed that those with less experience reported lower scores on culture of safety measures compared to their more experienced counterparts. The findings highlighted the differences of culture of safety reported by direct-care personnel versus their more senior counterparts in managerial roles. However, the researchers focused on healthcare personnel in general and were not specific about the role of nurses in the study. Furthermore, there was no evidence that OR personnel were included in the study. These results were consistent with a study conducted in the in the United Kingdom examining the perceived culture of safety between experienced surgeons and novice surgeons. The findings showed that the perceived culture of safety was higher in the more 
experienced group, which led the researchers to accept that a culture of safety was positively related to the years of clinical experience of personnel. Due to the variance in safety culture perceptions between personnel with varying lengths of clinical experience, the researchers suggested that safety culture surveys should encompass personnel of varying clinical experience to attain a more valid clinical picture of perceived safety (Bethune, Canter, \& Abrams, 2012).

In addition, a 2014 literature review of safety in healthcare illustrated that junior employees' lack of confidence based on insufficient experience was an impedance to speaking up for safety. The researchers concluded that physicians and nurses with limited clinical experience were reluctant to voice concerns over patient safety, even if they had awareness of the perils of such omissions (Okuyama, Wagner, \& Bijnen, 2014). This is a specific facet of clinical experience that may affect adverse patient outcomes, particularly in the OR setting where patients are typically unable to speak for themselves.

Similar to length of clinical experience, nurse engagement has also been linked to safety (Salanova, Lorens, Cifre, Martınez, \& Schaufeli, 2003). Keyko (2014) stated that the concept of nurse engagement has been a topic of focus stimulated primarily by factors such as the rates of medical error and quality of patient care. For example, a 2005 Gallup poll found nurse engagement to be a significant predictor of patient safety in the U.S. Studies have shown that engaged personnel show an aptitude for learning and have characteristics associated with proactive and safe behavior (Salanova et al., 2003; Sonnentag, 2003; Wadsworth, Felton \& Linus, 2016; Brooks Carton et al., 2019). These findings are consistent with the conclusion from Garcia-Sierra et al. (2016) who reported that nurse engagement affects how nurses perform, ultimately influencing patient 
outcomes. Furthermore, in a qualitative study that explored the barriers to nurse engagement, nurses articulated that being in an environment that was unsafe for patients led to burnout, which has been described as the opposite of engagement. This study's findings presented a positive relationship between nurse engagement and safe patient care (Freeney \& Tiernan, 2009).

While nurse engagement and length of clinical experience have been associated with safety in separate relationships, there is evidence that length of clinical experience and nurse engagement are closely aligned. For example, employees who have high levels of engagement display favorable dispositions towards their roles and organizations with which they are employed, and therefore they tend to remain with their organizations for longer periods (Demerouti et al., 2001; Schaufeli \& Bakker, 2004). In addition, the positive relationship between all three factors; nurse experience, nurse engagement, and safety was demonstrated in a 2013 study asserting that engaged nursing teams were more willing to remain in their positions longer, and they reported better quality of patient care (Van Bogaert, Wouters, Willems, Mondelaers, \& Clarke, 2013). Based on Benner's (1984) theory as well as the extant literature, it was hypothesized that length of clinical experience and nurse engagement are strong predictors of culture of safety.

\section{Research Design and Methods}

The study applied a non-experimental, correlational design in which the strength and associations of the relationships between the length of the OR nurses' clinical experience, OR nurse engagement, and OR culture of safety were investigated. 


\section{Sample}

A power analysis demonstrated that the number of participants required to detect $10 \%$ of the variance in culture of safety is 90 . Two types of sampling were employed. Purposeful sampling was applied to intentionally select nurses who currently work in an OR, and snowball sampling was applied in an effort to attain participants with various amounts of clinical experience that is consistent with the various stages of "novice to expert" as described by Benner (1984). Eligibility criteria included currently licensed registered nurses who worked primarily in an operating room setting for at least 20 hours per week, who were over the age of 18, and who were English-speaking.

\section{Recruitment}

Recruitment was conducted by email, verbal announcement, social media, and flyers. Eligible OR nurses were invited to participate by accessing an electronic link provided to them through each recruitment method. A group email was sent to the members of the local Association of periOperative Registered Nurses (AORN) chapter to inform the OR nurses about the study. The email contained the study purpose, information on eligibility criteria, and a request for participants. Announcements were made at the meetings at which OR nurses attend monthly, and members of the local AORN chapter were asked to distribute the flyers containing information about the study to other OR nurses. Furthermore, a social media platform was used to advertise the study, its purpose, and call for eligible participants. The social media site included the web link to allow participants to access the data collection forms. The survey was available for two months, including April and May 2019. 


\section{Instruments}

The study had three data collection tools: a demographic data collection form, the Safety Attitudes Questionnaire (SAQ) (Sexton et al. 2006), and the Utrecht Work Engagement Scale (UWES) (Schaufeli, Bakker, \& Salanova, 2006).

The demographic data form collected data on the following: length of overall nursing clinical experience measured in years; length of clinical nursing experience specifically in the OR measured in years; age measured in years; level of nursing education, which included diploma, associate degree, baccalaureate degree, and graduate degree; status of OR nurse certification (Certified Nurse in the Operating Room [CNOR] yes:no); and number of ORs within the department in which the nurse is employed.

The SAQ (Sexton et al., 2006) is a self-reported questionnaire developed to measure the safety attitudes of front-line workers. It is one of the most common tools to measure safety culture (Nguyen et al. 2015), and it has been used frequently to measure safety culture in the OR (Allard et al. 2011; Magill et al. 2017; Carvalho et al. 2015; Carney et al. 2010; St. Pierre, et al. 2017; Ongun \& Intepeler, 2017). The SAQ elicits an illustration of the safety culture by surveying employees' attitudes towards safety within their organization. It has been used to measure the culture of safety within organizations because the attitudes and creed of personnel related to safety reflects the culture of safety within the organization (Sexton et al., 2006; Singer et al., 2009). The instrument consists of seven dimensions that include teamwork climate, safety climate, job satisfaction, stress recognition, perceptions of hospital management, perceptions of unit management, and working conditions. The original instrument had 60 items (Sexton et al. 2006); however, it has been shortened to contain 41 items (Nguyen et al., 2015). Each item is answered 
using a five-point Likert scale as follows: Disagree Strongly, Disagree Slightly, Neutral, Agree Slightly, and Agree Strongly. The shortened survey total SAQ scale Cronbach's alpha was 0.85 , which showed good internal consistency, and the Cronbach's alpha of each subscale ranged between 0.70 and 0.86 (Nguyen, et al. 2015).

The UWES (Schaufeli, Bakker, \& Salanova, 2006) measured nurse engagement, which may be described as "a positive work-related state of fulfillment that is characterized by vigor, dedication, and absorption" (Schaufeli, Bakker, \& Salanova, 2006). Initially, the scale had 24 items; however, it was shortened to a 9-item version by the scale authors. The scale has seven answer options that include Never, Almost Never, Rarely, Sometimes, Often, Very Often, and Always. The UWES has three dimensions including vigor, described as a high level of energy and mental resilience at work; dedication, described as a substantial involvement in one's work that is accompanied by enthusiasm and a sense of significance; and absorption, described as full immersion in one's work with difficulties detaching from it (Schaufeli, Bakker, \& Salanova, 2006). Cronbach's alpha of the shortened UWES scales 0.92, which showed good internal consistency, and the Cronbach's alpha of the three subscales ranged between 0.77 and 0.85 (Schaufeli, Bakker, \& Salanova, 2006).

\section{Procedure}

IRB approval was obtained prior to the study. The quantitative tools, the demographic data collection form, the SAQ (Sexton et al., 2006) and the UWES (Schaufeli, Bakker, \& Salanova, 2006), were used to collect data. Data were collected and managed using REDCap (Research Electronic Data Capture) electronic data capture tools hosted at the University of Louisville (Harris et al. 2009). REDCap is a secure, web- 
based application designed to support data capture for research studies, providing 1) an intuitive interface for validated data entry; 2) audit trails for tracking data manipulation and export procedures; 3) automated export procedures for seamless data downloads to common statistical packages; and 4) procedures for importing data from external sources.

The REDCap link to connect to the surveys was available through social media for a period of two months. A copy of the link was on a group email that was sent to AORN members and on flyers provided to OR nurses. When participants clicked on the provided REDCap link, they were presented with the data collection platform that was preceded with a consent preamble. Following the consent preamble, the participants addressed the demographic data collection form followed by the UWES, and the SAQ surveys, which offered Likert scale answer options. The participants were given the opportunity to submit their answers at the conclusion of the final form. At the conclusion of data collection, the data from REDCap was exported into Excel and then uploaded into SPSS 25.0 (IBM Corp., 2017).

Survey participants had the opportunity to voluntarily enter into a gift card drawing as a study incentive by providing their name and either a phone number or email address (unattached from the survey data) directly to the researcher. After the survey closed, three $\$ 50$ gift cards were awarded by a drawing with the names of those who submitted their contact information. Entry into the drawing was not required to complete the survey.

\section{Data Management and Analysis}

Data were analyzed using SPSS Version 25.0 (IBM Corp, 2017). Descriptive analysis was conducted to describe the participants' demographic data, including length 
of overall nurse experience, length of OR nurse experience, age, level of nursing education, number of ORs in department of employment, and OR nurse certification status. Frequencies, percentages, means, standard deviations, median, mode, and range were calculated, depending on the level of the data. Using Cronbach's alpha, the internal consistency of the SAQ was .958, and the internal consistency of the UWES was .904, which indicated strong internal consistency of both scales. The statistical significance for all analyses was based on the conventional level of significance of 0.05 .

The independent variables for the study included length of OR nurses' clinical experience and OR nurse engagement, which were continuous variables. The dependent variable was culture of safety in the OR, which was also a continuous variable.

The SAQ was answered using a five-point Likert scale ranging from 1 "Disagree Strongly" to 5 "Agree Strongly". An additional option 6 indicated "not applicable." Prior to analysis, all responses of 6 were explored and characterized as missing data because the value did not contribute to the true SAQ score. Missing data was managed by applying the "exclude cases pairwise" option in SPSS, which excluded the participant only if they were missing the data required for the specific analysis being conducted.

Three of the items on the SAQ were reverse coded. No manipulation of coding was required for the UWES items. The sum scores for both the SAQ and for the UWES were calculated. The assumptions of the dependent variable, culture of safety, were evaluated. The assumption of normality was met as evidenced by non-statistically significant Kolmogorov-Smirnov test. The assumption of homoscedasticity was assessed by examining a scatterplot. A VIF tolerance statistics was computed to assess multicollinearity between the length of clinical experience and nurse engagement, using 
greater than or equal to five as a cutoff, with a result of 1.01 , which did not indicate multicollinearity.

For Aim 1, the following bivariate associations were examined: the length of the OR nurses' experience and OR culture of safety $(\mathrm{H} 1)$, length of the OR nurse engagement and OR culture of safety (H2), and length of the OR nurses' experience and OR nurse engagement. For Aim 2, a standard multiple linear regression in which all of the variables were entered into the model simultaneously was conducted to examine the strength and association of the relationships between OR culture of safety, OR nurse engagement, and length of OR nurse clinical experience. For Aim 3, the adjusted $\mathrm{R}^{2}$ was computed to determine the amount of variance in culture of safety attitudes that was explained by the model. Standardized beta coefficients were assessed to determine which variable, length of clinical experience or nurse engagement, appeared to be the strongest predictor of culture of safety. In addition, the interaction between the independent variables, length of OR clinical experience and OR nurse engagement was explored. For Aim 4, an independent sample t-test was conducted to assess if there were differences in culture of safety scores between those who had a certification in OR nursing (CNOR) versus those who had not.

\section{Results}

\section{Demographic Characteristics}

The majority of participants $(\mathrm{N}=96)$ had baccalaureate degrees $(52.4 \%)$, and less than half (42.6\%) had CNOR status (Table 7). The ages of the participants ranged from 21 to 70 years old with a mean age of $43.79(\mathrm{SD}=12.8)$. The length of OR nurse experience ranged from less than 1 year to 49 years, $(M=3.82 ; S D=12.32)$ years, although the mean 
the length of experience as a nurse in general was $18.61(\mathrm{SD}=13.05)$ years. The number of ORs within the departments of where the participants were employed ranged from 2 to $25(\mathrm{M}=11.74 ; \mathrm{SD}=6.17)$.

Table 8

Descriptive Characteristics of Participants $(\mathrm{N}=96)$

Characteristic M (SD)

Age in years

$43.79(12.83)$

Length of experience as a nurse

$18.61(13.05)$

Length of experience in the OR

Number of ORs-mean

$11.74(6.17)$

Frequency (\%)

CNOR

Yes

$40(42.6)$

No

$54(57.4)$

Education Level

Diploma

Associates degree

Baccalaureate degree

$52(54.2)$

Graduate degree

$16(16.7)$

Note. $\mathrm{M}=$ mean; $\mathrm{SD}=$ standard deviation 
Overall, the mean culture of safety score (SAQ) was 155.23 (SD 30.42) (Table 8). Scores ranged from 65 to 198 with lower scores indicating a lower culture of safety. Scores for the UWES averaged 45.09 (SD 7.91) with a range of 21 to 63; lower scores represented lower engagement.

Table 9

Descriptive Statistics for the SAQ and UWES

\begin{tabular}{lcccc}
\hline Variable & $\mathrm{N}$ & $\mathrm{M}(\mathrm{SD})$ & Min-Max & $\begin{array}{c}\text { Possible } \\
\text { Range }\end{array}$ \\
\hline SAQ & 63 & $155.23(30.42)$ & $65-198$ & $41-205$ \\
UWES & 93 & $45.09(7.91)$ & $21-63$ & $9-63$
\end{tabular}

Note. $\mathrm{M}=$ mean; $\mathrm{SD}=$ standard deviation; Min-Max= minimum and maximum score values

\section{Relationships between length of the OR nurses' clinical experience and culture of safety attitudes in the OR}

Bivariate associations between the length of OR nurse experience, OR nurse engagement, and OR safety culture using Pearson correlation technique indicated that although length of OR nurse experience was positively correlated with culture of safety in the OR, the relationship was weak and not statistically significant $(\mathrm{r}=.168 ; p=.189)$ (Table 9). Similarly, the length of OR nurse experience had a weak relationship with OR nurse engagement, and the relationship was not statistically significant $(\mathrm{r}=.105 ; p=.32)$. This is a contrast however to the relationship between OR nurse engagement and OR culture of safety in which the result of the Pearson correlation concluded that there was a strong, positive significant relationship $(\mathrm{r}=.628 ; p=<.001)$. 
Table 10

Bivariate Associations between Culture of Safety, OR Nurse Work Engagement, and Length of OR Nurse Experience ( $\mathrm{N}=93$ )

\begin{tabular}{lccc}
\hline & $\begin{array}{c}\text { Culture of Safety in } \\
\text { the OR }\end{array}$ & $\begin{array}{c}\text { OR Nurse Work } \\
\text { Engagement }\end{array}$ & $\begin{array}{c}\text { Length of OR Nurse } \\
\text { Experience }\end{array}$ \\
\hline $\begin{array}{l}\text { Culture of Safety in } \\
\text { the OR }\end{array}$ & 1.0 & $.628^{*}$ & .168 \\
$\begin{array}{l}\text { OR Nurse Work } \\
\text { Engagement }\end{array}$ & $.628^{*}$ & 1.00 & .105 \\
$\begin{array}{l}\text { Length of OR Nurse } \\
\text { Experience }\end{array}$ & .168 & .105 & 1.00 \\
\hline
\end{tabular}

\section{Predictors of OR culture of safety}

OR nurse engagement predicted culture of safety in the OR $(p=<.001)$, while the length of OR nurse experience did not $(p=>0.05)$. Out of the two independent variables, OR nurse engagement made the strongest unique contribution to predicting culture of safety in the OR (Table 10).

\section{Percent of variance in culture of safety explained by length of OR nurses' clinical experience and $O R$ nurse engagement}

Both independent variables, the length of OR nurse experience and OR nurse engagement, explained $38 \%$ (adjusted $\mathrm{R}^{2}=0.385$ ) of the variance in OR culture of safety, and the amount of variance explained was statistically significant $(p=<.001)$. OR nurse engagement was shown to predict OR culture of safety, such that as nurse engagement increased by one unit, the culture of safety increased by 2.37 units, while controlling for the length of nurse experience, and this association was statistically significant $(p<.001)$.

\section{Table 11}

Associations between OR Culture of Safety, Length of OR Nurse Experience, and OR Nurse Engagement $(\mathrm{N}=96)$ 


\begin{tabular}{lccccc}
\hline Variable & $\begin{array}{c}\text { Unstandardized } \\
\text { coefficient } \beta\end{array}$ & $\begin{array}{c}\text { Standardized } \\
\text { coefficient } \beta\end{array}$ & $\mathrm{p}$-value & $\begin{array}{c}\text { Semi-partial } \\
\text { Correlations }\end{array}$ & Collinearity (VIF) \\
\hline $\begin{array}{l}\text { Length of OR } \\
\text { Nurse }\end{array}$ & -.001 & -.052 & .542 & -.051 & 1.013 \\
Experience & & & & & \\
OR Nurse & -.086 & -.594 & $.000^{*}$ & -.591 & 1.013 \\
Engagement & & & & &
\end{tabular}

$* p<.05$

Table 12

Summary of Predictors of OR Culture of Safety $(\mathrm{N}=96)$

\begin{tabular}{lccccc}
\hline Variable & $\beta$ & SE & Beta & $\mathrm{t}$ & $\mathrm{p}$ \\
\hline Constant & 44.64 & 17.76 & & 2.51 & 0.02 \\
OR Nurse Engagement & 2.37 & 1.00 & 0.62 & 6.11 & $<.001^{*}$ \\
Length of OR Nurse Experience & 0.25 & 0.112 & 1.02 & 1.02 & 0.313 \\
\hline
\end{tabular}

$* p<.05$

\section{Differences in OR culture of safety based on CNOR}

Those with CNOR certification had a higher mean culture of safety score

$(M=166.43 ;$ SD 25.71) than those who did not hold a CNOR certification (M=145.06; SD

31.16). This difference was statistically significant $(\mathrm{t}=2.97 ; \mathrm{df}=61 ; p=.004)$.

\section{Discussion}

Achieving and sustaining a culture of safety in the OR is a topic of relevance because the OR is laden with risks due to its information-intensive, complex nature that, increases the vulnerability of OR patients. It is imperative, therefore, to examine factors associated with and predictive of a culture of safety in the OR. This study is the first to specifically examine OR nurse characteristics, such as OR nurse length of experience as well as OR nurse engagement and their associations with OR culture of safety. 
The findings of the study demonstrated that OR nurse engagement has a strong association with and is a significant predictor of OR culture of safety. However, as this study used the sum scores of the UWES and the SAQ for analysis, further research is warranted to examine the relationships between the dimensions of each of the instruments to better understand relationships and associations between specific aspects of engagement and safety.

The overall conclusion that nurse engagement is correlated with safety is consistent with the extant literature (Salanova et al., 2003; Sonnentag, 2003; Brooks Carthon, et al., 2019). However, previous studies linking nurse engagement with safety were not conducted with OR nurse participants, and none of the studies linking nurse engagement to safety applied the UWES as the instrument to measure nurse engagement.

The length of OR nurse experience was also shown to be positively correlated with OR culture of safety. However, the relationship was weak and not statistically significant. This finding is generally inconsistent with previous studies that demonstrated strong positive associations between length of nurse experience and safety (Singer, et al., 2009; Bethune, Canter, \& Abrams, 2012; Riley, Dearmon, Mestas, \& Buckner, 2016). In addition, the findings related to the OR nurse length of experience are incongruent with Benner's (1984) Novice to Expert theory, which suggests that as nurses gain experience in their roles, their ability to make sound, safe clinical decision-making is enhanced.

Similarly, the relationship between OR nurse length of experience and OR nurse's engagement was not statistically significant, which is an indication that length of clinical experience is not required to be engaged in one's work. The findings led to a rejection of hypothesis 1 (There is a positive relationship between the length of the OR nurses' 
experience and OR culture of safety); hypothesis 2 (There is a positive relationship between the length of the OR nurses' experience and OR nurse engagement), and hypothesis 4 (length of the OR nurses' experience and nurse engagement predicts OR culture of safety). However, the study finding accepts hypothesis 2 (There is a positive relationship between OR nurse engagement and OR culture of safety).

While the study results favored of OR nurse engagement over the length of OR nurse experience in relation to an OR culture of safety, managers should not assuage efforts to promote retention of their nurses, and arguably, the length of OR nurse experience is more likely beneficial when it is paired with OR nurse engagement.

Furthermore, participants with CNOR certification demonstrated higher culture of safety scores than those without CNOR certification. However, the study did not determine if having CNOR certification was a predictor of OR culture of safety. Therefore, more research is warranted to examine the specific role CNOR certification plays in safety and engagement.

\section{Limitations}

As previously noted, the SAQ and the UWES each include multiple sub-scales. However, in this study, scores for the SAQ and for the UWES were summed and the subscales were not addressed. Future research should explore relationships between the instruments sub-scales. A second limitation relates to the accessibility of the survey. Although every effort was made to recruit participants from a variety of geographic areas, data on participant's location was not gathered. Therefore, it is unknown if the participants represented a wide geographic area or were primarily located in the Louisville, Kentucky bi-state region. Similarly, because of the use of social media 
platform for recruitment, access to the survey was not restricted to US residents.

Therefore, data generated from other countries could limit the generalizability of the findings to US hospitals specifically.

With the ubiquitous nature of cell phones, it is possible that participants completed the surveys on their phones. This increases the challenge of visualizing the entire context of the study instruments on a relatively small screen. This may have hampered accuracy when answering questions with Likert-type responses, as with the UWES and SAQ instruments. It is possible that this factor played a role in the selection of the sixth response on the SAQ, which represented a "not applicable" answer, and it was not possible to determine if participants intended to choose the "not applicable" response or if participants intended to choose the "always" response, which was the fifth option. All responses consisting of 6 were subsequently assigned as "missing" data, to avoid artificially inflating the SAQ scores. This action reduced the sample size of the participants who completed the SAQ to 63. Finally, self-reported scales have limitations because they are subject to individual participant emotions and ability to interpret the questions.

\section{Conclusions}

The results illustrated that direct measures should be taken to increase OR nurse engagement so that a culture of safety can be fostered and sustained in the OR. Wadsworth, Felton, and Linus (2016) suggested that leadership personnel have a responsibility to engage their employees, and these authors provided concrete examples on how to engage nurses. Strategies to engage nurses included fortifying strategic plans that align nurses with their organization and involve direct-care nurses in shared-decision 
making. They also suggested the "SOAR" (strengths, opportunities, aspirations, and results) model, which has been shown to effectively encourage innovation and nurture engagement of direct-care employees. In addition, Riley et al. (2016) reported that efforts such as applying a shared governance model was an effective strategy to engage and empower employees. Rather than attaining input only from managerial personnel, the inclusion of direct-care nurses in the overall organization mission can result in significant advancement and improvement in clinical quality and patient safety (Wadsworth et al., 2016; Brooks Carthon et al., 2018). Furthermore, Demsey and Assi (2018) promulgated the crucial link that managerial staff have over the work environment and nurse engagement, which ultimately leads to higher patient safety standards and outcomes.

While extant literature provides strategies to increase nurse engagement, there is little information on engaging OR nurses specifically. Therefore in light of the study findings and the unique nature of OR nurses, further research is warranted to explore specific strategies on engaging OR nurses. Furthermore, while this study focused on two specific OR nurse characteristics, studies examining other factors that may predict an OR culture of safety would be worthy. 


\section{CHAPTER V SYNTHESIS AND CONCLUSIONS}

This dissertation served to: (1) identify the primary safety concerns in the operating room (OR) from the perspective of practicing OR nurses; (2) explore the concept of normalization of deviance in the OR and identify perceived barriers and facilitators to adhering to safety standards; and, (3) investigate the strength and association of the relationships between the culture of safety in the OR, the length of the OR nurses' clinical experience, and OR nurse engagement. This chapter synthesizes the research findings from both the qualitative and quantitative studies. Finally, implications for further research, clinical practice, and policy are presented and discussed.

\section{Synthesis}

A prolific interest in organizational culture of safety was generated in the 1990s, which was in part due to a culmination of well-publicized global disasters that occurred in the late 1980s, such as the National Aeronautical Space Administration (NASA) Challenger explosion, the Chernobyl nuclear disaster, the Exxon Valdez oil spill, and the London King's Cross Underground fire (Pidgeon, 1998). Pidgeon (1988) suggested that safety culture was a pertinent consideration within high-risk organizations because if safety culture is lacking, working conditions set the stage for system failures. The exploration of global disasters has led researchers to conclude that system failures arise from a significant period of inattention to detail and a normalization of deviance (Gerstein \& Ellsberg, 2008; Pidgeon, 1998; Vaughan, 1996). 
Vaughan (1996) explained that normalization of deviance is a concept whereby individuals and teams deviate from acceptable standards of conduct to the point where the deviation becomes the new norm. The concept is particularly pervasive when there is a period of absence from adverse events, which assuages the perception of risk. This is pertinent for safety culture research because etiolated attitudes towards safety violations in the workplace are antecedents to adverse events (Pidgeon, 1998; Rundmo, 1996; Price \& Williams, 2018). Normalization of deviance can be particularly concerning in the OR, because it is a complex, information-intensive field in which patient safety is highly dependent on staff behavioral norms that are consistent with a culture of safety (Wright, 2016; Link, 2019).

A culture of safety is critical to thwart errors in the OR, and therefore, it is imperative to examine the factors that support a culture of safety and those that may attenuate it. The identification and acknowledgement of patient safety threats and antecedents to adverse events should continue to be a salient provocation in healthcare and play a role in guiding safety improvement strategies (Vincent, Carthey, Macrae, \& Amalberti, 2017). Singer et al. (2009) concluded that a culture of safety within a healthcare facility was associated with higher levels of healthcare personnel safetyrelated behaviors and better patient outcomes, which established a link between a healthcare organization's culture of safety and patient safety. It is essential to investigate OR personnel practice and safety culture, which is increasingly being recognized as an important strategy and a necessary precursor to improving the widespread deficits in patient safety (Pronovost \& Sexton, 2005; Vincent, et al., 2017).

Examining safety, however, is not unidimensional. While social sciences have 
typically measured culture of safety by examining individual attitudes towards safety, other sciences, such as engineering, have traditionally assessed safety with a focus on the examination of systems (Pidgeon, 1998). Vincent et al. (2017) reported that organizations have historically leaned towards incident reports and investigative efforts to assess safety; however, adding assessments that include the anticipation of safety risk in the future is becoming widely accepted (Vincent, et al., 2017). Therefore, it was appropriate to combine more than one approach to exploring OR safety, whereby specific safety practices are explored within the tapestry of safety culture so that risk can be identified and mitigated before adverse events occur.

This mixed-methods study, whereby the qualitative component is referred to as Phase 1 and the quantitative component is referred to as Phase 2, presented a unique approach to examining safety culture in the OR. Safety practices and normalization of deviance in the OR were explored qualitatively as well as quantitatively by examining OR safety culture and factors that may affect it. Such factors include length of OR nurse experience and OR nurse engagement.

\section{Methods}

The mixed methods study used an exploratory sequential design in three-phases. Phase 1 included the collection of qualitative data, Phase 2 included the collection of quantitative data, and Phase 3 included interpretation of data from the prior phases (Creswell \& Plano Clark, 2018). The exploratory sequential design is illustrated in Figure 3.

Phase 1 applied a focused ethnographic approach, which may be defined as a subsidiary of traditional ethnography whereby particular elements of a culture are 
examined against the background of a larger culture (Wolf, 2010). This approach was appropriate to use for this study because safety behaviors and normalization of deviance were explored in OR nurses, who are a subculture of nurses at large.

Phase 2 applied a non-experimental, correlational design in which the strength and associations of the relationships between OR culture of safety, the length of OR nurses' experience, and OR nurse engagement were investigated. The final phase involved the summarization and interpretation of both qualitative and quantitative results in a discussion on how qualitative results may be tested and quantitative results may be generalized.

\section{Figure 2}

Exploratory Sequential Design (Creswell \& Plano Clark, 2018)

Phase 1

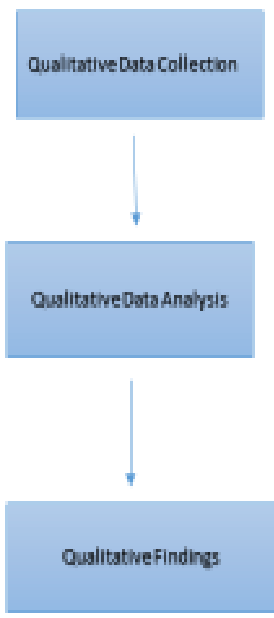

Phase 2
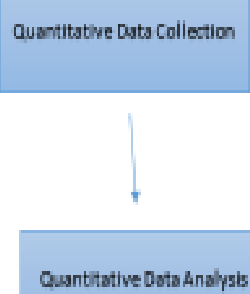

Quantitative OstaAnalysis

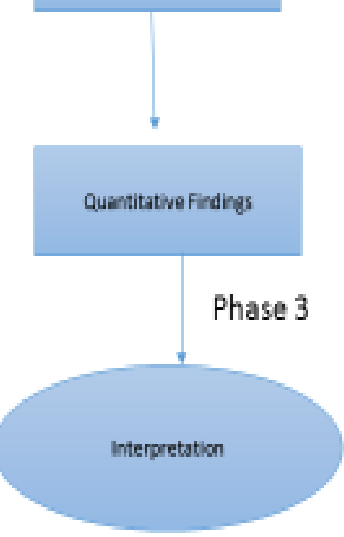




\section{Results for Phase 1}

Aim 1) OR nurses reported areas of primary safety concerns as follows: There were two primary themes identified including productivity pressures and inadequate staff to support workload. Additional concerns that were reported included "infobesity;" inconsistency of order entry, and general complacency of staff. Aim 2) Specific examples of normalization of deviance in the OR were illustrated, for example, participants indicated that OR nurses acquire a general complacency in relation to the adherence of safety standards, particularly when there is a perceived absence of adverse events. Aim 3) Themes relating to the barriers to adhering to safety standards and reasons for normalization of deviance included: productivity pressures, generalized complacency, complacency related to length of experience, social pressures, and negative acculturation. An additional reason included disconnection from direct consequences; however, this was not categorized as a theme because there was only one supporting quote.

Facilitators to adhering to safety standards and protective factors against normalization of deviance included nurse engagement and supportive managerial relationships with OR nurses. Nine out of ten participants indicated that nurse engagement supported the adherence to safety standards. Engagement was also associated with confidence and empowerment, and nurses alluded that clinical experience was not a precursor for engagement. In addition, three out of ten participants reported that their relationship with managerial personnel was supportive. Participants inferred that a supportive relationship with management led to open communication and a safer environment because managers understood the challenges in the OR and supported OR 
nurses' work. In addition, participants indicated that supportive managerial relationships contributed to OR nurse engagement.

\section{Results for Phase 2}

The results for Phase 2 demonstrated that the length of OR nurse experience was positively correlated with culture of safety in the OR; however, the relationship was weak, and it was not statistically significant. In contrast, OR nurse engagement had a statistically significant relationship with culture of safety, and it was a significant predictor of OR culture of safety. Finally, certified OR nurses had significantly higher culture of safety scores compared with those without certification.

\section{Integration of the Results from both Phase 1 and Phase 2}

Data integration is central to mixed methods analysis. The integration of the Phase 1 and Phase 2 findings involved the summarization and interpretation of both qualitative and quantitative results focusing on how qualitative results may be tested and how quantitative results may be generalized (Creswell and Plano Clark, 2018).

OR safety concerns, normalization of deviance in the OR, and perceived barriers and facilitators to adhering to safety standards were uncovered and categorized. Reported barriers were heavily associated with an insensitivity to the importance of safety-related protocols and a normalization of deviance. While barriers and facilitators to the adherence to safety protocols were uncovered, there was a specific focus on the length of OR nurse experience and OR nurse engagement.

Phase 1 results, particularly from the perspective of OR nurse engagement and the length of OR nurse experience, informed Phase 2. The specific focus on OR nurse engagement and the length of OR nurse experience is key to the integration of Phase 1 
and Phase 2 because OR nurse participants from Phase 1 reported that the length of OR nurse experience was often associated with a lax attitude toward safety, whereas it was reflected that OR nurse engagement was associated with sound clinical decision making ability that was consistent with patient safety. Although a pilot qualitative study that was conducted one year earlier did not include specific questions on length of experience or nurse engagement, these factors also emerged from the pilot study findings as influencers over safety practices.

Phase 1 findings corroborated those of Phase 2, which inferred that the relationship between the length of OR nurse experience and OR culture of safety was weak and not statistically significant. In contrast, OR nurse engagement had a positive, statistically significant relationship with culture of safety in the OR. Table 12 illustrates the joint display of the integration of the results from both Phase 1 and Phase 2 of this mixed-methods study.

Table 13

Joint Display to Represent Linked Results for the Exploratory Sequential Design

\begin{tabular}{|l|c|c|c|l|}
\hline $\begin{array}{l}\text { Qualitative } \\
\text { theme }\end{array}$ & $\begin{array}{c}\text { Quantitative } \\
\text { Independent } \\
\text { Variable }\end{array}$ & $\begin{array}{c}\text { Quantitative } \\
\text { Dependent } \\
\text { Variable }\end{array}$ & $\boldsymbol{p}$ & Mixed Methods Interpretation \\
\hline $\begin{array}{l}\text { OR nurse } \\
\text { engagement } \\
\text { is associated } \\
\text { with } \\
\text { protection } \\
\text { against } \\
\text { normalization } \\
\text { of deviance }\end{array}$ & $\begin{array}{c}\text { OR Nurse } \\
\text { Engagement } \\
\text { OR Culture } \\
\text { of Safety }\end{array}$ & $<.001$ & $\begin{array}{l}\text { OR nurses suggested that } \\
\text { engagement was a characteristic } \\
\text { that protected nurses against } \\
\text { normalization deviance and } \\
\text { provided them with the ability to } \\
\text { withstand pressures to deviate } \\
\text { from the meticulous safety } \\
\text { standards required to ensure } \\
\text { safety for OR patients. } \\
\text { Quantitative results supported this } \\
\text { finding by demonstrating a } \\
\text { statistically significant } \\
\text { relationship between OR nurse }\end{array}$ \\
\hline
\end{tabular}




\begin{tabular}{|c|c|c|c|c|}
\hline $\begin{array}{l}\text { Qualitative } \\
\text { theme }\end{array}$ & $\begin{array}{c}\text { Quantitative } \\
\text { Independent } \\
\text { Variable }\end{array}$ & $\begin{array}{c}\text { Quantitative } \\
\text { Dependent } \\
\text { Variable }\end{array}$ & $p$ & Mixed Methods Interpretation \\
\hline & & & & $\begin{array}{l}\text { engagement and OR culture of } \\
\text { safety. Also, Phase } 2 \text { results } \\
\text { indicated that OR nurse } \\
\text { engagement was a statistically } \\
\text { significant predictor of OR } \\
\text { culture of safety. }\end{array}$ \\
\hline $\begin{array}{l}\text { Length of } \\
\text { OR nurse } \\
\text { experience is } \\
\text { related to } \\
\text { complacently } \\
\text { resulting in } \\
\text { normalization } \\
\text { of deviance }\end{array}$ & $\begin{array}{c}\text { Length of OR } \\
\text { Nurse } \\
\text { Experience }\end{array}$ & $\begin{array}{l}\text { OR Culture } \\
\text { of Safety }\end{array}$ & .542 & $\begin{array}{l}\text { Most OR nurse participants } \\
\text { indicated that length of OR nurse } \\
\text { experience was a reason for } \\
\text { normalization deviance because } \\
\text { nurses can become lax and } \\
\text { complacent over time and } \\
\text { unwilling to change behavior } \\
\text { based on updated policies and } \\
\text { procedures. Also, the length of } \\
\text { OR nurse experience was not } \\
\text { significantly correlated with OR } \\
\text { culture of safety, nor was it a } \\
\text { significant predictor of OR } \\
\text { culture of safety. }\end{array}$ \\
\hline
\end{tabular}

\section{Research Implications}

This study is the first to explore normalization of deviance in the OR, and while abundant information was gleaned, the OR remains a fertile environment for further research that explores safety practices as well as additional factors that support or attenuate a culture of safety. In light of the prominent theme of productivity pressure both as a safety concern and as a prevailing reason for normalization of deviance, more research is needed on the impact of productivity pressure on the quality of care in the OR.

While efficiencies and effective time management strategies are warranted for 
fiscal responsibility, particularly in light of the revenue-generating position that most ORs hold in their facilities, the extent that excessive productivity pressure is placed on direct-care OR nurses is harmful and detrimental to patient safety. There is an abundance of literature relating to OR efficiencies with fiscal motives embedded (Fong, Smith, \& Langerman, 2016; Rothstein \& Raval, 2018); however, there is a dearth of information on the non-monetary price that the quest for excessive efficiencies exacts. Therefore, strategies to improve efficiencies without hampering OR quality of care should be examined and highlighted.

While rich data related to the reasons for normalization of deviance were gathered from Phase 1, it is clear that that more research should be done to explore additional reasons. For example, one participant said “We don't ever see any positioning injury [data]. None of that is brought to our attention, like when we have monthly meetings, none of that is shared with staff at all." This quote was stated in reference to OR nurses being lulled in to a sense of complacency because of a lack of awareness of OR adverse events. While the quote refers specifically to positioning injury, the same concept applies to other adverse events that can occur in the OR without knowledge of such at the time of its occurrence, such as surgical site infection, and the retainment of foreign objects. The OR nurse participant suggested that it would be safer to have transparent conversations between management and direct-care nurses in relation to adverse events. This concept should be explored further because the lack of awareness or knowledge of adverse events can assuage the perception of risk and contribute to normalization of deviance. Research on potential barriers to the open discussion of such events, for example, litigious issues or fear of facility defamation, should be explored. 
A prominent finding from both phases includes the salience of OR nurse engagement to support safety practices and the sustenance of an OR safety culture. Therefore, strategies for increasing OR nurse engagement should be explored and tested. In addition, areas for future research include examining the relationships between the specific dimensions of the SAQ and UWES scales, which could offer an insight into the associations between OR nurse engagement and OR culture of safety. For instance, two of the dimensions of the SAQ include, perceptions of hospital management and perceptions of unit management; therefore the examination of these dimensions specifically in relation to predictors, such as engagement or other nurse characteristics may offer more knowledge on how management plays a role in safety.

Synthesis of Phase 1 and 2 study results indicated that the length of OR nurse experience can be a barrier to the adherence to safety standards and is not related to an OR culture of safety. However, this finding is inconsistent with the findings of other studies that reflected that length of nurses' experience was related to patient safety (Benner, 1984; Singer, et al., 2009; Bethune, Canter, \& Abrams, 2012; Riley, Dearmon, Mestas, \& Buckner, 2016). While previous studies examining the realtionship between length of experience and patient saftey were not based on OR nurses, further research on the value of length of nurse experience within various practice settings may be warranted. In addition, the discrepancy of study findings in relation to length of experience may point to an inherent difference in OR nurses compared to nurses within other clinical practice subspecialties, which opens an opportunity for further research.

Finally, Phase 2's findings showed that OR nurses who have CNOR certification had significantly higher OR culture of safety scores compared with their non-certified 
peers; however, it is unclear if certification is predictive of an OR safety culture. Thus research is warranted to examine the specific role CNOR certification plays in OR safety.

\section{Practice Implications}

The exclusive focus on OR nurses in the study bears much relevance to OR clinical practice because of the unique, prominent, and multi-faceted roles that they assume. For example, the responsibilities of the OR nurse include, but are not limited to: preparing the operating room with supplies and equipment for the intended surgery, interviewing and assessing the surgical patient prior to surgery, supporting the anesthesia provider with the administration of anesthesia and placement of endotracheal tube, assisting with the positioning of the patient, performing the surgical skin preparation, ensuring functioning equipment, overseeing the surgery to ensure safety is maintained, helping the scrub personnel, and, caring for the patient in the immediate postoperative period. Therefore, while there are many roles within the surgical team, the OR nurse is the only team member who manages the many different facets of care over the entire perioperative period. Each task that is required of OR nurses carries risk, and if the tasks are performed without adhering wholly to safety standards, the vulnerability for surgical patients increases exponentially.

The findings of Phase 1 illustrated a pervasiveness of normalization of deviance, and therefore, it is imperative to acknowledge and disseminate these findings to OR personnel and management so that the reasons for such as be assuaged. The primary reasons for normalization of deviance included: productivity pressure, generalized complacency, complacency related to length of experience, social pressures, and negative acculturation. Each of these reasons need to be addressed openly and in detail so that 
these barriers to adhering to safety standards can be mitigated to foster a culture of OR safety.

The responsibility for providing safety to surgical patients lies with all perioperative team members, nurse educators, managers, and administrators. Patient safety needs to be at the forefront of all discussions related to practice and policy. An open, transparent culture should exist so that OR nurses do not encounter barriers to the adherence to safety standards in the workplace without being supported by managerial personnel.

It is crucial that a culture of safety in the OR is created, fostered, and sustained. OR nurses should be given enough support and resources to meet the complex demands of the role as well as to effectively balance productivity, efficiency and safety. Complacency towards safety standards should be reported in a non-punitive manner. In addition, an awareness of the high-risk nature of the OR should be promoted frequently so that complacency is seen as an outlier more than the norm. Social pressures related to incivility that may exist in the workplace need to be rooted out by providing opportunities to report incivility without fear of retribution. In addition, encouragement of team members to support each other as safety practices are conducted is essential. Negative acculturation should be managed with help from nurse educators who can formally outline orientation practices to help standardize orientation of new OR nurses. The adherence to safety standards should be modeled by preceptors so that safety practice deviations that can reinforce unsafe practices in preceptees are deemed unacceptable. As OR nurse engagement emerged as a facilitator to the adherence to safety standards, and well as its demonstration of being a significant predictor of an OR safety 
culture, implementing strategies to increase OR nurse engagement gains prime importance. In addition, supportive managerial relationships also emerged as a facilitator to adherence to safety practices. Furthermore, Phase 1 participants indicated that supportive managerial relationships facilitated safety standards adherence, and they inferred that supportive relationships increased nurse engagement. The link between managerial support and safety and engagement is supported in the literature (Demsey \& Assi, 2018; Price \& Williams, 2018; Harvey \& Sotardi, 2017; Wadsworth, Felton, and Linus, 2016). Therefore, relationships between OR nurses and managerial personnel need to be fortified and energized with respect, understanding, and other managerial behaviors fostering a supportive workplace, which nurtures a safe work environment and accommodates nurse engagement (Dempsey \& Assi, 2018).

There are additional benefits to involving OR leaders in the management of safety. For example, Price and Williams (2018) concluded that leadership involvement had a pertinent role in patient safety. In addition, while the mixed methods study focused solely on direct-care OR nurses, it is imperative to avoid allocating blame and criticism to any one person or group because opportunities to improve safety standards lie beyond those who provide direct care in the OR. Thus, there needs to be a shift in focus from individual guilt to overall systems and processes (Price \& Williams, 2018).

\section{Policy Implications}

Policies should be implemented that fortify a system of adverse events and nearmiss reporeting within the OR. While there is documented evidence that adverse events occur in the OR (Steelman, Shaw, Shine, \& Hardy-Fairbanks, 2019), the reporting of such is ambiguous and poorly understood. This is primarily due to challenges with the 
reporting of data due to lack of transparency and the lack of standardized national reporting systems and definitions (Thiels et al., 2015). Specifically, prospectively collected data on the incidence of surgical never events are limited, and many studies involve voluntary reporting to external agencies with innate bias (Austin \& Pronovost, 2015; Theils et al. 2015). This is problematic because the lack of comprehensive data may not accurately present the reality of flawed safety systems. Therefore, greater attention needs to be given to standardized reporting systems relating to OR adverse events, near-misses, and general OR safety standard deviation in a non-punitive and supportive environment (Harvey \& Sotardi, 2017). Policies related to the fortification of reporting systems should promote transparency and the acknowledgement of near-misses and deviations so that risk can be attenuated before deviations from safety standards become entrenched and normalized in personnel's practice behavior.

In addition, policies should be implemented to ensure that that there is a sharedgovernance approach within hospital facilities. This model would help establish an inclusion of direct-care OR nurses in decision-making related to OR practices, which is a strategy that would promote engagement in OR nurses (Harvey \& Sotardi, 2017; Brook Carthon et al., 2019). Furthermore, Harvey and Sotardi (2018) suggested that when managers attain feedback from direct-care employees, an effective conduit is created to better understand challenges, such as productivity pressures, that may exist in the directcare environment. In this way, a more transparent, safer culture can be enhanced.

\section{Summary}

Achieving and sustaining a culture of safety in the OR is worthy of significant attention because the environment is laden with risk due its information-intensive, 
complex nature, which increases vulnerability for OR patients. It is imperative, therefore, to examine the safety concerns of OR nurses, uncover practices consistent with normalization of deviance, identify barriers and facilitators to adhering to OR safety protocols as well as examine the relationships of factors associated with the culture of safety within the OR.

Normalization of deviance is embedded within the OR culture but it has not widely acknowledged because of its stealth nature. Considering the mixed-methods study findings, it is essential to highlight the pervasive nature of this phenomenon within ORs. Efforts should be made to disseminate the findings so that a platform of openconversation and transparency between OR nurses and their managerial counterparts can be formed. Acknowledging barriers to safety practices, providing opportunities for the involvement of OR nurses in decision-making related to OR processes as well as openly discussing needed resources for safe and efficient practice may foster a culture of safety in the OR.

Finally, OR nurse engagement and supportive managerial relationships with OR nurses were identified as facilitators that guide OR nurses to practice safely as well as contribute to an overall culture of safety. Therefore, it is imperative to promote OR nurse engagement and supportive managerial relationships with OR nurses as strategies to improve the adherence to safety standards and mitigate risk for vulnerable surgical patients. 


\section{REFERENCES}

Allard, J., Bleakley, A., Hobbs, A., \& Coombes, L. (2011). Pre-surgery briefings and safety climate in the operating theatre. British Medical Journal Quality and Safety, 20(8), 711-717. doi:10.1136/bmjqs.2009.032672.

Amalberti, R., Vincent, C., Auroy, Y., \& de Saint Maurice, G. (2006). Violations and migrations in health care: a framework for understanding and management. Quality and Safety in Health Care, 15 (1), 66-71. doi:10.1136/qshc.2005.015982

Austin, J. M. \& Pronovost, P. J. (2015). Never events and the quest to reduce preventable harm. The Joint Commission Journal on Quality and Safety, 41(6), 279-288.

Balkin, R. S., \& Kleist, D. M. (2017). Counseling research; A practitioner-scholar approach. Alexandria, VA: American Counseling Association.

Banja, J. (2010). The normalization of deviance in healthcare delivery. Business Horizons, 53(2), 139. doi:10.1016/j.bushor.2009.10.006.

Banschbach, S. K. (2009). Revisiting the universal protocol. AORN Journal, 89(2), 257259. doi:10.1016/j.aorn.2009.01.001.

Bethune, R., Canter, R., \& Abrams, P. (2012). What do surgical trainees think about patient safety culture, and is this different from their consultants? Clinical Risk, 18, 52-57. doi:10.1258/cr.2012.011043.

Benner, P. (1984). From novice to expert: Excellence and power in clinical nursing practice. Menlo Park, CA: Addison-Wesley. 
Brooks Carthon, J. M., Hatfield, L., Plover, C., Dierkes, A., Davis, L., Hedgeland, T., Sanders, A.M., Visco, F., Holland, S., Ballinghoff, J. DelGuidice, M., Aiken, L. H. ( 2019). Association of nurse engagement and nurse staffing on patient safety. Journal of Nursing Care and Quality, 34(1), 40-46.

Carney, B. T., West, P., Neily, J., Mills, P. D., \& Bagian, J. P. (2010). The effect of facility complexity on perceptions of safety climate in the operating room: Size matters. American Journal of Medical Quality, 25(6), 457-461. doi:10.1177/1062860610368427.

Carthey, J. (2014). What's in my buckets today? Foreseeing and forestalling patient harm. J Perioperative Practice, 24(3), 40-44.

Carthey, J., Walker, S., Deelchand, V., Vincent, C., \& Griffiths, W. H. (2011). Breaking the rules: understanding non-compliance with policies and guidelines. $B M J, 343$, d5283. doi:10.1136/bmj.d5283.

Carvalho, P., Gottems, L. B., Pires, M., de Oliveira, M. L. (2015). Safety culture in the operating room of a public hospital in the perception of healthcare professionals. Rev. Latin American Enfermagem. 23 (6), 1041-1048

Christian, C. K., Gustafson, M. L., Roth, E. M., Sheridan, T. B., Gandhi, T. K., Dwyer, K., Dierks, M. M. (2006). A prospective study of patient safety in the operating room. Surgery, 139(2), 159-173. doi:10.1016/j.surg.2005.07.037.

Collins, S. J., Newhouse, R., Porter, J., \& Talsma, A (2014). Effectiveness of the surgical safety checklist in correcting errors: A literature review applying Reason's Swiss cheese model, AORN Journal, 100(1), 65-79. 
Cramm, J. M. \& Nieboer, A. P. (2019). Acculturation is associated with older Turkish immigrants'self-management abilities. BMC Public Health, 19(1228, 1-9. doi: 10.1186/s12889-019-7471-0.

Creswell, J. W., \& Plano Clark, V. L. (2018). Designing and Conducting Mixed Methods Research (Third ed.). Thousand Oaks, CA: SAGE Publications, Inc.

Dedoose. Los Angeles, CA: SocioCultural Research Consultants, LLC www.dedoose.com.

Dekker, S. (2011). Patient safety: A human factors approach. Boca Raton, FL: CRC Press.

Demerouti, E., Bakker, A. B., de Jonge, J., Janssen, P. P., \& Schaufel, W. B. (2001). Burnout and engagement at work as a function of demands and control. Scandinavian Journal for Work, Environment \& Health, 27, 279-286.

Demsey, C. \& Assi, M. J. (2018). The impact of nurse enagagement on quality, safety, and the experience of care. What nurse leaders need to know. Nursing Administration Quarterly, 43(3), 278-283.

Espin, S., Lingard, L., Baker, G. R., \& Regehr, G. (2006). Persistence of unsafe practice in everyday work: an exploration of organizational and psychological factors constraining safety in the operating room. Quality and Safety in Health Care, 15(3), 165-170. doi:10.1136/qshc.2005.017475.

Fong, A. J., Smith, M., \& Langerman, A. (2016). Efficiency improvement in the operating room. Journal of Surgical Research, 204 (2), 371-383. 
Freeney, Y. M., \& Tiernan, J. (2009). Exploration of the facilitators of and barriers to work engagement in nursing. International Journal of Nursing Studies, 46(12), 1557-1565. doi:10.1016/j.ijnurstu.2009.05.003.

Garcia-Sierra, R., Fernandez-Castro, J., \& Martinez-Zaragoza, F. (2016). Work engagement in nursing: an integrative review of the literature. Journal of Nursing Management, 24(2), 101-111. doi:10.1111/jonm.12312.

Gerstein, M. S., \& Ellsberg, M. (2008). Flirting with disaster : why accidents are rarely accidental. New York: Union Square Press.

Gerstle, C. (2018). Parallels in saftey between aviation and healthcare. Journal of Pediatric Surgery, 53, (5), 875-878.

Goldenberg, M. E. \& Elterman, D. (2019). From box ticking to the black box: The evolution of operating room saftey. World Journal of Urology. DOI: 10.1007/s00345-019-02886-5.

Harris, P. A., Taylor, R. Thielke, R., Payne, J. Gonzalez, N., \& Conde, J. G.(2009) Research electronic data capture (REDCap) - A metadata-driven methodology and workflow process for providing translational research informatics support. Journal of Biomedical Informatics, 42(2), 377-381.

Harvey, H. B. \& Sotardi, S. T. (2017) Normalization of deviance and practical drift. Journal of the American College of Radiology, 14, (12), 1572-1573.

IBM Corp. Released 2017. IBM SPSS Statistics for Windows, Version 25.0. Armonk, NY: IBM Corp.

IOM. (2000). Crossing the Quality Chasm: A New Health System for the 21 st Century. Washington, DC: The National Academies Press. 
IOM. (2006). Preventing Medication Errors. Washington, D.C.: The National Academies Press.

Kaye, A. D.,McDowell, J. L., Diaz, J.H., Buras, J. A., \& Young, A. E. (2015). Effective strategies in improving operating room case delays and cancellations at an academic medical center. The Journal of Medical Practice Management, 30(6), 24-29.

King, C. A. (2010). To err is human, to drift is normalization of deviance. AORN Journal, 91(2), 284-286. doi:10.1016/j.aorn.2009.10.020.

Keyko, K. (2014). Work engagement in nursing practice: a relational ethics perspective. Nursing Ethics, 21(8), 879-889. doi:10.1177/0969733014523167

Keyko, K., Cummings, G. G., Yonge, O., \& Wong, C. A. (2016). Work engagement in professional nursing practice: A systematic review. International Journal of Nursing Studies, 61, 142-164. doi:10.1016/j.jpnurstu.2016.06.003

Kohn, L. T., Corrigan, J. M., \& Donaldson, M. S. (2000). To Err is Human: Building a Safer Health System. Retrieved from Washington (DC).

Lepanluoma, M., Takala, R., Kotkansalo, A., Rahi, M., \& Ikonen, T. S. (2014). Surgical safety checklist is associated with improved operating room safety culture, reduced wound complications, and unplanned readmissions in a pilot study in neurosurgery. Scandinavian Journal of Surgery, 103(1 ), 66-72. doi:10.1177/1457496913482255.

Link, T. (2019). Guideline implementation: A safe environment of care. AORN Journal, $110(2), 170-176$. 
Magill, S. T., Wang, D. D., Rutledge, C., Lau, D., Berger, M. S., Sankaran, S., Imershein, S. G. (2017). Changing operating room culture: Implementation of a postoperative debrief and improved safety culture. World Neurosurgery, 107, 597-603.

Maslow, A. (1954). Motivation and personality. New York, NY: Harper.

McNamara, S. A. (2011). The normalization of deviance: What are the perioperative risks? AORN Journal, 93 (6), 796-801.

Mehtsun, W. T., Ibrahim, A. M., Diener-West, M., Pronovost, P. J., \& Makary, M. A. (2013). Surgical never events in the United States. Surgery, 153(4), 465-472. doi:10.1016/j.surg.2012.10.005.

Nguyen, G, Gambashidze, N., Ilyas, S. A., \& Pascu, D. (2015). Validation of the safety attitudes questionnaire (short form 2006) in Italian in hospitals in the northeast of Italy, BMC Health Services Research, 15(284), 1-8.

Odom-Forren (2011). The normalization of deviance: a threat to patient safety. Journal of Perianesthesia Nursing, 26(3), 216-219. doi:10.1016/j.jopan.2011.05.002.

Okuyama, A., Wagner, C., \& Bijnen, B. (2014). Speaking up for patient safety by hospital-based health care professionals: a literature review. BMC Health Services Research, 14(61),1-8.

Ongun, P., \& Intepeler, S. S. (2017). Operating room professionals' attitudes towards patient safety and the influencing factors. Pakistan Journal of Medical Science, 33(5), 1210-1214. doi:10.12669/pjms.335.13615.

Patton, M. Q. (1990). Qualitative Evaluation and Reserach Methods (Second ed.). Newbury Park, CA: SAGE Publications. 
Peters, J. A., Young, D., White, J., \& Mahal-van Brenk, C. (2013). Managing OR revenue under new payment models. Healthcare Financial Management, 67(4), 108-112, 114, 116.

Pidgeon, N. (1988) Safety culture: key theoretical issues. Work and Stress, 12(3), 202216.

Price, M. R. \& Williams, T. C. (2018). When doing wrong feels so right: Normalization of deviance. Journal of Patient Safety, 2 (1), 1-2.

Prielipp, R. C., Magro, M., Morell, R. C., \& Brull, S. J. (2010). The normalization of deviance: do we (un)knowingly accept doing the wrong thing? AANA Journal, 78(4), 284-287.

Pronovost, P. \& Sexton, B. (2005). Assessing safety culture guidelines and recommendations. Quality of Safety in Health Care, 14(4), 231-233.

Rasmussen, J. B. (1997). Risk management in a dynamic society: A modelling problem. Safety Science, 27(2), 183-213.

Reason, J. T. (1990). Human Error, Cambridge, England: Cambridge University Press.

Reid, J. H. (2014). Violations and migrations perioperative practice: how organisational drift puts patients at risk. Journal of Perioperative Practice, 24(3), 45-49.

Richards, L., \& Morse, J. M. (2013). Readme First for a User's Guide to Qualitiative Methods (Third ed.). Thousand Oaks, CA: SAGE Publications Inc.

Riley, B. H., Dearmon, V., Mestas, L., \& Buckner, E. B. (2016). Frontline Nurse Engagement and Empowerment: Characteristics and Processes for Building Leadership Capacity Nursing Administration Quarterly, 40(4), 325-333. doi:10.1097/NAQ.0000000000000186. 
Rothstein, D. \& Raval, M. V. (2018). Operating room efficiency. Seminars in Pediatric Surgery, 27, 79-85.

Salanova, M., Llorens, S., Cifre, E., Martınez, I., \& Schaufeli, W. B. (2003). Perceived collective efficacy, subjective well-being and task performance among electronic work groups: an experimental study. Small Group Research, 34, 43-73.

Schaufeli, W., \& Bakker, A. B. (2004). Job demands, job resources, and their relationship with burnout and engagement: a multi-sample study. Journal of Organizational Behavior, 25, 293-315.

Schaufeli, W., Salanova, M., Gonzalez-Rom, V., \& Bakker, A. (2002). The measurement of engagement and burnout: a two sample confirmatory factor analytic approach. Journal of Happiness Studies, 3, 71-92.

Schaufeli, W. B., Bakker, A. B., \& Salanova, M. (2006). The measurement of work engagement with a short questionnaire; A cross-national study. Educational and Psychological Measurement, 66(4), 701-716. doi:0.1177/0013164405282471

Sexton, J. B., Helmreich, R. L., Neilands, T. B., Rowan, K., Vella, K., Boyden, J., Thomas, E. J. (2006). The Safety Attitudes Questionnaire: psychometric properties, benchmarking data, and emerging research. BMC Health Services Research, 6, 44. doi:10.1186/1472-6963-6-44.

Simmons, D., Symes, L., Guenter, P., \& Graves, K. (2011). Tubing misconnections: normalization of deviance. Nutr Clin Pract, 26(3), 286-293. doi:10.1177/0884533611406134 
Singer, S., Lin, S., Falwell, A., Gaba, D., \& Baker, L. (2009). Relationship of safety climate and safety performance in hospitals. Health Services Research, 44(2), 399-421. doi:10.1111/j.1475-6773.2008.00918.x

Sonnentag, S. (2003). Recovery, work engagement, and proactive behavior: a new look at the interface between non-work and work Journal of Applied Psychology, 88, 518-528.

St.Pierre, M., Gall, C., Breuer, G., \& Schüttler, J. (2017). Does annual simulation training influence the safety climate of a university hospital? : Prospective 5-year investigation using dimensions of the safety attitude questionnaire. Anaesthesist. doi:10.1007/s00101-017-0371-8

Stahel, P. F., Mehler, P. S., Clarke, T. J., \& Varnell, J. (2009). The 5th anniversary of the "Universal Protocol": pitfalls and pearls revisited. Patient Safety in Surgery, 3(1), 14. doi:10.1186/1754-9493-3-14.

Steelman, V. M., Shaw, C., Shine, L., Hardy-Fairbanks, A. J. (2019). Unintentionally retained foreign objects: A descriptive study of 308 sentinel events and contributing factors. The Joint Commission Journal of Quality and Patient Safety, $45,245-249$.

The Joint Commission, (2004). Universal Protocol. Retrieved from https://www.jointcommission.org/standards_information/up.aspx website:

Theils, C. A., Lal, T. M., Niewow, J. M., Pasupathy, K. S., Blocker, R. C., Aho, J. M. Morgenthaler, T. I., Cima, R. R. Hallback, S \& Bingener, J. (2105). Surgical never events and contributing human factors, Surgery, 158 (2), 515-521. 
Ulrich, B., \& Kear, T. (2014). Patient safety and patient safety culture: Foundations of excellent healthcare delivery. Nephrology Nursing Journal, 41(5), 447-456.

Van Bogaert, P., Wouters, K., Willems, K., Mondelaers, M., \& Clarke, S. (2013). Work engagement supports nurse workforce stability and quality of care: nursing teamlevel analysis in psychiatric hospitals. Journal of Psychiatric and Mental Health Nursing, 20, 679-686.

Vaughan, D. (1996). The Challenger launch decision:Risky technology, culture, and deviance at NASA. Chicago: University of Chicago Press.

Vaughan, D. (1997). The trickle down effect: Policy decisions, risky work, and the challenger tragedy. California Management Review, 39 (2), 80-102.

Vincent, C. \& Amalberti, R. (2015). Safety in helathcare is a moving target. British Medical Journal for Quality and Saftey, 24, 539-540.

Vincent, C. Carthey, J., Macrae, C., \& Amalberti, (2017). Safety analysis over time: Seven major changes to adverse event investigation. Implementation Science, 12 (151), 1-10. doi: 10.1186/s13012-017-0695-4

Wadsworth, B., Felton, F., \& Linus, R. (2016). SOARing into strategic planning: Engaging nurses to achieve significant outcomes. Nursing Administration Quarterly, 40 (4), 299-306.

Walker, L. O. \& Avant, K. C. (2019).Strategies for Theory Construction in Nursing (6 ${ }^{\text {th }}$ edition). New York, NY: Pearson.

Wilbanks, B. A., Geisz-Everson, M. Clayton, B. A. \& Boust, R. R. (2018). Transfer of care in perioperative settings: A descriptive qualitative study. AANA Journal, 86(5), 401-407. 
Wolf, Z. R. (2010). Ethnography: The Method. In P. L. Munhall (Ed.), Nursing Research: A Qualitative Persective (5th ed., pp. 315). Sudbury, MA: Jones \& Bartlett Learning.

Wright, M. I. (2016). Implementing no interruption zones in the perioperative environment. AORN Journal, 104(6), 536-540. doi:10.1016/j.aorn.2016.09.018.

Wright, M. I. (2018). Safety-related practices in the operating room. Midwest Nursing Research Society 42nd Annual Research Conference, Cleveland, OH (Poster presentation, April 4, 2018).

Zahiri, H. R., Stromberg, J., Skupsky, H., Knepp, E. K., Folstein, M., Silverman, R., \& Singh, D. (2011). Prevention of 3 "never events" in the operating room: fires, gossypiboma, and wrong-site surgery. Surgical Innovation, 18(1), 55-60. doi:10.1177/1553350610389196. 


\section{CURRICULUM VITAE}

NAME: Margaret Imelda Wright

ADDRESS: 9000 Hurstwood Court

Louisville, KY 40222

DOB: Killarney, Co. Kerry, Ireland- January 20, 1971

\begin{tabular}{|c|c|c|}
\hline \multicolumn{3}{|c|}{ EDUCATION } \\
\hline 2019 & University of Louisville, Louisville, Kentucky & $\mathrm{PhD}$ (Nursing) \\
\hline 2015 & McKendree University, Louisville, Kentucky & MSN (Nursing) \\
\hline 2008 & McKendree University, Louisville, Kentucky & BSN (Nursing) \\
\hline 1992 & $\begin{array}{l}\text { Catherine McAuley School of Nursing, } \\
\text { Mercy Hospital, Cork, Ireland. }\end{array}$ & Diploma (Nursing) \\
\hline \multicolumn{3}{|c|}{ CERTIFICATIONS/LICENSES } \\
\hline \multicolumn{2}{|c|}{ 2008-present } & (CNOR) \\
\hline $1996-$ & Registered Nurse, Kentucky RN1 & 34521 \\
\hline
\end{tabular}

PROFESSIONAL MEMBERSHIPS

2001- Present Association of periOperative Registered Nurses (AORN)

2018-Present Midwestern Nursing Research Society (MNRS)

PROFESSIONAL POSITIONS

2016-present Assistant Professor

School of Nursing

University of Louisville

Louisville, KY 
2008- 2016

2002-2008

1996-2001

1994-1995

1992-1994
Education Coordinator

Staff Development

Clinical Informatics RN

Operating Room Circulator

Endoscopy RN

Assistant Manager Endoscopy

Medical Surgical Staff RN
Surgical Services

Baptist Health Louisville

Louisville, KY

Main Operating Room Dept.

Baptist Health Louisville Louisville, KY

Endoscopy Department Baptist Health Louisville

Louisville, KY

Endoscopy Department King's College Hospital, London, UK

Mercy Hospital

Cork, Ireland

\section{PUBLICATIONS}

1. Wright, M. I. (2016). Implementing no interruption zones in the perioperative environment. AORN Journal, 104(6), 536-540.Thomas, M., Roberts, H, Williams, S., Wright, M. I., Cronin, S.N, \& Odom-Forren, J. (2014). Examining the effectiveness of controlled breathing, with or without aromatherapy, in the treatment of postoperative nausea in female patients undergoing outpatient laparoscopic procedures. Journal of Perianesthesia Nursing, 29 (5), 42-48.

3. Wright, M. I. (2011). Peripheral nerve blocks in the outpatient surgery setting. AORN Journal, 94(1), 59-74.

4. Wright, M. I. (2007). Cerebral aneurysm; treatment and perioperative nursing care. AORN Journal, 85(6), 1172-1182.

5. Wright, M. I. (1987). Esophageal varices; treatment and implications. Gastroenterology Nursing, 21(1) 2-5.

\section{PRESENTAITONS AT LOCAL SYMPOSIA}

1. Wright, M. I. (2018, September). Safety-related practices in the Operating Room. Research Louisville, Louisville, KY 
2. Wright, M. I. (2018, March). Safety-Related Practices in the Operating Room. Graduate Student Regional Research Conference (GSRRC), Louisville, KY.

3. Wright, M. I. (2017, September). Patients' Perceptions of Bedside Shift Report. Research Louisville, Louisville, KY.

4. Wright, M. I. (2016, April). Implementing the No Interruption Zone in the Perioperative Environment. AORN meeting, Baptist Health Louisville, Louisville, KY.

5. Wright, M. I (2015, March). Examining the effectiveness of controlled breathing, with or without aromatherapy, in the treatment of postoperative nausea in female patients undergoing outpatient laparoscopic procedures. Annual Research Symposium, Baptist Health Louisville, Louisville, KY

6. Wright, M. I. \& Thomas, M. (2014, September). Examining the effectiveness of controlled breathing, with or without aromatherapy, in the treatment of postoperative nausea in female patients undergoing outpatient laparoscopic procedures. Research Louisville, Jewish Hospital, Louisville, KY.

7. Wright, M. I. (2010, March). Improving Patient Outcomes Following Interscalene Blocks for Arthroscopic Shoulder Surgery. Annual Research Symposium, Baptist Health Louisville, Louisville, KY

\section{POSTER PRESENATIONS}

1. Wright, M. I. (2019, April). Safety-related practices in the Operating Room. AORN Global Surgical Conference and Expo, Nashville, TN.

2. Wright, M. I. (2018, September). Safety-related practices in the Operating Room. Research Louisville, Louisville, KY

3. Wright, M. I. (2018, April). Safety-related practices in the Operating Room. MNRS, Cleveland, $\mathrm{OH}$.

4. Wright, M. I. (2017, September). Patients' Perceptions of Bedside Shift Report. Research Louisville, Louisville, KY.

5. Wright, M. I. (2015, October). Implementing the No Interruption Zone (NIZ) in the Perioperative Environment. Louisville Nursing Research Symposium, Norton Hospital, Louisville, KY.

6. Wright, M. I. (2015, September). Implementing the No Interruption Zone (NIZ) in the Perioperative Environment. KONL $11^{\text {th }}$ Annual Leadership Conference. 
7. Wright, M. I. \& Thomas, M. (2014, September). Examining the effectiveness of controlled breathing, with or without aromatherapy, in the treatment of postoperative nausea in female patients undergoing outpatient laparoscopic procedures. Research Louisville, Jewish Hospital, Louisville, KY.

8. Wright, M. I. (2010, March). Improving Patient Outcomes Following Interscalene Blocks for Arthroscopic Shoulder Surgery

\section{PRESENTATIONS REALTED TO STAFF DEVELOPMENT}

1. Wright, M. I. (2015, July). Implementing the No Interruption Zone (NIZ) in the Perioperative Environment. Baptist Health Louisville, Louisville, KY.

2. Wright, M. I. (2015, June). Surgical Counts. Baptist Health Louisville, Louisville, KY.

3. Wright, M. I. (2015, June). The Universal Protocol in the Perioperative Environment. Baptist Health Louisville, Louisville, KY.

4. Wright, M. I. (2012, June). Fire Safety in the OR. Baptist Health Louisville, Louisville, KY.

AWARDS

- Voted as faculty favorite 2016-2017

- Voted as faculty favorite $2017-2018$

- Voted as faculty favorite 2018-2019

- Voted as BSN Program Outstanding Faculty member 2018-2019

\section{PROFESSIONAL ACTIVITY}

Served as an expert witness for attorney groups relating to malpractice defense in operating room on separate occasions:

- July- November 2017

- January - March 2018

- May 2018- Present

\section{VOLUNTARY ACTIVITY}

- Surgery on Sunday

- Kentucky Refugee Ministries

- Kentuckiana AORN 